\title{
Discovery of Candidate H2O Disk Masers in Active Galactic Nuclei and Estimations of Centripetal Accelerations
}

\section{Citation}

Greenhill, Lincoln J., Paul T. Kondratko, James M. Moran, and Avanti Tilak. 2009.

"Discovery of Candidate H2O Disk Masers in Active Galactic Nuclei and Estimations of Centripetal Accelerations." The Astrophysical Journal 707 (1) (November 25): 787-799. doi:10.1088/0004-637x/707/1/787.

\section{Published Version}

doi::10.1088/0004-637X/707/1/787

\section{Permanent link}

http://nrs.harvard.edu/urn-3:HUL.InstRepos:26519245

\section{Terms of Use}

This article was downloaded from Harvard University's DASH repository, and is made available under the terms and conditions applicable to Other Posted Material, as set forth at http:// nrs.harvard.edu/urn-3:HUL.InstRepos:dash.current.terms-of-use\#LAA

\section{Share Your Story}

The Harvard community has made this article openly available.

Please share how this access benefits you. Submit a story.

Accessibility 


\title{
DISCOVERY OF CANDIDATE $\mathrm{H}_{2} \mathrm{O}$ DISK MASERS IN ACTIVE GALACTIC NUCLEI AND ESTIMATIONS OF CENTRIPETAL ACCELERATIONS
}

\author{
Lincoln J. Greenhill, Paul T. Kondratko ${ }^{1}$, James M. Moran, and Avanti Tilak \\ Harvard-Smithsonian Center for Astrophysics, 60 Garden St., Cambridge, MA 02138, USA; greenhill@ cfa.harvard.edu \\ Received 2009 June 23; accepted 2009 October 27; published 2009 November 25
}

\begin{abstract}
Based on spectroscopic signatures, about one-third of known $\mathrm{H}_{2} \mathrm{O}$ maser sources in active galactic nuclei (AGNs) are believed to arise in highly inclined accretion disks around central engines. These "disk maser candidates" are of interest primarily because angular structure and rotation curves can be resolved with interferometers, enabling dynamical study. We identify five new disk maser candidates in studies with the Green Bank Telescope, bringing the total number published to 30. We discovered two (NGC 1320, NGC 17) in a survey of 40 inclined active galaxies $\left(v_{\text {sys }}<20,000 \mathrm{~km} \mathrm{~s}^{-1}\right)$. The remaining three disk maser candidates were identified in monitoring of known sources: NGC 449, NGC 2979, and NGC 3735. We also confirm a previously marginal case in UGC 4203. For the disk maser candidates reported here, inferred rotation speeds are $130-500 \mathrm{~km} \mathrm{~s}^{-1}$. Monitoring of three more rapidly rotating candidate disks (CG 211, NGC 6264, VV 340A) has enabled measurement of likely orbital centripetal acceleration, and estimation of central masses $\left((2-7) \times 10^{7} M_{\odot}\right)$ and mean disk radii $(0.2-0.4 \mathrm{pc})$. Accelerations may ultimately permit estimation of distances when combined with interferometer data. This is notable because the three AGNs are relatively distant $\left(10,000 \mathrm{~km} \mathrm{~s}^{-1}<v_{\text {sys }}<15,000 \mathrm{~km} \mathrm{~s}^{-1}\right)$, and fractional error in a derived Hubble constant, due to peculiar motion of the galaxies, would be small. As signposts of highly inclined geometries at galactocentric radii of $\sim 0.1-1 \mathrm{pc}$, disk masers also provide robust orientation references that allow analysis of (mis)alignment between AGNs and surrounding galactic stellar disks, even without extensive interferometric mapping. We find no preference among published disk maser candidates to lie in high-inclination galaxies. This provides independent support for conclusions that in late-type galaxies, central engine accretion disks and galactic plane orientations are not correlated.
\end{abstract}

Key words: galaxies: active - galaxies: Seyfert - ISM: molecules - masers

\section{INTRODUCTION}

Extragalactic $\mathrm{H}_{2} \mathrm{O}$ maser emission from active galactic nuclei (AGNs) in spiral galaxies in some cases traces highly inclined disk structures at radii $\sim 0.1-1 \mathrm{pc}$ from the central engines. Cases established by direct interferometric imaging that resolves position-velocity structure include NGC 4258 (e.g., Miyoshi et al. 1995; Argon et al. 2007), NGC 1068 (Greenhill \& Gwinn 1997), NGC 3079 (Yamauchi et al. 2004; Kondratko et al. 2005), Circinus (Greenhill et al. 2003b), UGC 3789 (Reid et al. 2009), and NGC 6323 (Braatz et al. 2007). These "disk masers" have common spectroscopic characteristics, highly redand blueshifted emission lines, called "high-velocity" emission lines, that more or less evenly bracket the systemic velocity, and emission close to the systemic velocity, called "low-velocity" emission lines. Both are defined in the frame of the rotating disk, projected along the line of sight. We use this terminology of "high" and "low" velocity lines throughout this paper. This template is also observed in other $\mathrm{H}_{2} \mathrm{O}$ masers for which imaging data are not available, and it can be a powerful diagnostic. In total, at least 30 published masers (out of 107 known) share these characteristics (e.g., Greenhill et al. 2008, and references therein). The high-velocity red- and blueshifted emission has been observed to be offset on the order of $10^{2}-10^{3} \mathrm{~km} \mathrm{~s}^{-1}$, among sources. We note that very long baseline interferometric (VLBI) imaging is essential to confirm the identity of disk masers. Hence, we have used the terminology "disk maser candidates" for sources with spectroscopic identification only. We also note that VLBI imaging of some disk masers shows signs of additional maser emission arising in outflows (e.g., Circinus) and jets (e.g., NGC 1068).

\footnotetext{
${ }^{1}$ Current address: Goldman Sachs, London, UK
}

$\mathrm{H}_{2} \mathrm{O}$ disk masers highlight structures oriented edge-on, or nearly so, and in close proximity to central engines. This is plausible because large reservoirs of molecular gas along lines of sight are required to generate detectable emission (Elitzur 1982). Among all $\mathrm{H}_{2} \mathrm{O}$ masers in AGNs, $95 \%$ exhibit X-ray absorption column densities $>10^{23} \mathrm{~cm}^{-2}$ (Greenhill et al. 2008). Among disk masers, the fraction is nearly as high, and the distribution of obscuring column densities peaks above $10^{24} \mathrm{~cm}^{-2}$. For thin disks, maser emission is beamed narrowly about the midplane, into a solid angle $\ll 4 \pi$, as dictated by requisite velocity coherence along lines of sight (e.g., Miyoshi et al. 1995). Geometry limits observable emission to disks that are highly inclined. Although common association has been well recognized with optical type- 2 and partly obscured type- 1 objects (e.g., Braatz et al. 1997b), disk maser emission is a sharper indicator of (inclination) orientation for nuclear structure than optical classifications are. In addition, it is a more direct indicator than, e.g., models of $\mathrm{Fe} \mathrm{K} \alpha$ emission from hot accretion disks (Nandra et al. 1997), the differential brightness of radio jets, and models of kiloparsec-scale ionization structures. Comparisons of inclination complement analyses of position angles and naturally enable analyses of relative three-dimensional orientation (Clarke et al. 1998; Pringle et al. 1999; Nagar \& Wilson 1999).

We conducted a survey of AGNs to detect new disk masers, selecting galaxies with inclined stellar disks (inclination angle $i>70^{\circ}$ ). We detected two disk maser candidates and one unclassified maser (Section 3.1). We also conducted deep integrations to identify indicators of disk maser emission among previously known sources, detecting another three (Section 3.2), and monitored another three known disk maser candidates to measure centripetal acceleration due to disk rotation (Section 3.3). We discuss estimation of black hole masses and disk radii in 
Section 4 as well as the distribution of inclinations among disk maser candidate hosts and misalignment between central engines and galactic stellar disks.

\section{OBSERVATIONS}

The survey was conducted during 2004-2005 with the Green Bank Telescope (GBT) of the NRAO, ${ }^{2}$ using two $200 \mathrm{MHz}$ intermediate frequency (IF) bands configured to overlap by $50 \mathrm{MHz}$, in each polarization. We tuned the center of the resulting $350 \mathrm{MHz}$ instantaneous bandwidth to the systemic velocity of each target source $\left(350 \mathrm{MHz} \sim 4880 \mathrm{~km} \mathrm{~s}^{-1}\right.$ for a representative recessional velocity of $5000 \mathrm{~km} \mathrm{~s}^{-1}$, assuming the optical definition of Doppler shift). The spectrometer delivered a channel spacing of $24.4 \mathrm{kHz}$. The dedicated observation of known $\mathrm{H}_{2} \mathrm{O}$ maser sources was conducted between 2003 and 2006 with the same setup, except for galaxy CG 211 (2003 December 8), where the two IFs were configured to overlap by $20 \mathrm{MHz}$ and the IF at the lower velocity was centered on the systemic velocity of the galaxy.

To obtain total-power spectra, we nodded the telescope by $3^{\prime}$ every 2 minutes between two positions on the sky so that each target was always present in the on-axis or off-axis beam. System temperatures $\left(T_{\text {sys }}\right)$ were measured against a calibrated noise source injected at the receiver and ranged from 23 to $75 \mathrm{~K}$ depending on elevation and weather conditions. To calibrate the spectra to flux density units, we used the gain curve obtained by the GBT staff based on measurements of opacitycorrected antenna temperature for NGC 7027 at $\sim 1.4 \mathrm{~cm}$ (R. Maddalena 2008, private communication). By comparing maser line amplitudes among beams and polarizations, we estimate that the calibration of the $T_{\mathrm{sys}}$ is accurate to within $30 \%$, an uncertainty that dominates the error budget for the flux density scale (and is probably due to calibration uncertainties for the noise sources). In comparison, antenna pointing corrections were obtained every $\sim 30$ minutes and were typically $<6^{\prime \prime}$, which corresponds to a loss less than $8 \%$ in source flux density for a $36^{\prime \prime}$ beamwidth (FWHM) at $1.3 \mathrm{~cm}$. Additional losses due to pointing errors induced by varying wind conditions were less than $20 \%$, which we estimate using the error model of Condon (2003) and the bottom $90 \%$ of measured wind speeds at GBT weather station 2, for each observation. (Our estimated losses are conservative, considering that wind speeds on the feed arm itself were typically lower.)

Data were reduced using custom scripts written in the Interactive Data Language. We subtracted a running boxcar average of width $6.25 \mathrm{MHz}$ to remove systematic baseline structure from the total-power spectra, excluding velocity intervals with signal that was detectable above the structure seen in raw integrations. The resulting $1 \sigma$ noise levels attained in an integration of $\sim 30$ minutes and corrected for atmospheric opacity estimated from tipping scans (from 0.03 to 0.2 ) were $4-14 \mathrm{mJy}$ in a $24.4 \mathrm{kHz}$ channel. The spectra presented here have been Hanning smoothed (multiple times) to a resolution of $108 \mathrm{kHz}$, though channel spacing of $24.4 \mathrm{kHz}$ is shown in the figures.

\section{RESULTS}

\subsection{Survey: Three New Masers}

In a survey of 40 inclined $\left(i>70^{\circ}\right)$ AGNs with $v_{\text {sys }}<18,000 \mathrm{~km} \mathrm{~s}^{-1}$ selected from the NASA/IPAC Extra-

\footnotetext{
2 The National Radio Astronomy Observatory is operated by Associated Universities Inc., under cooperative agreement with the National Science Foundation.
}
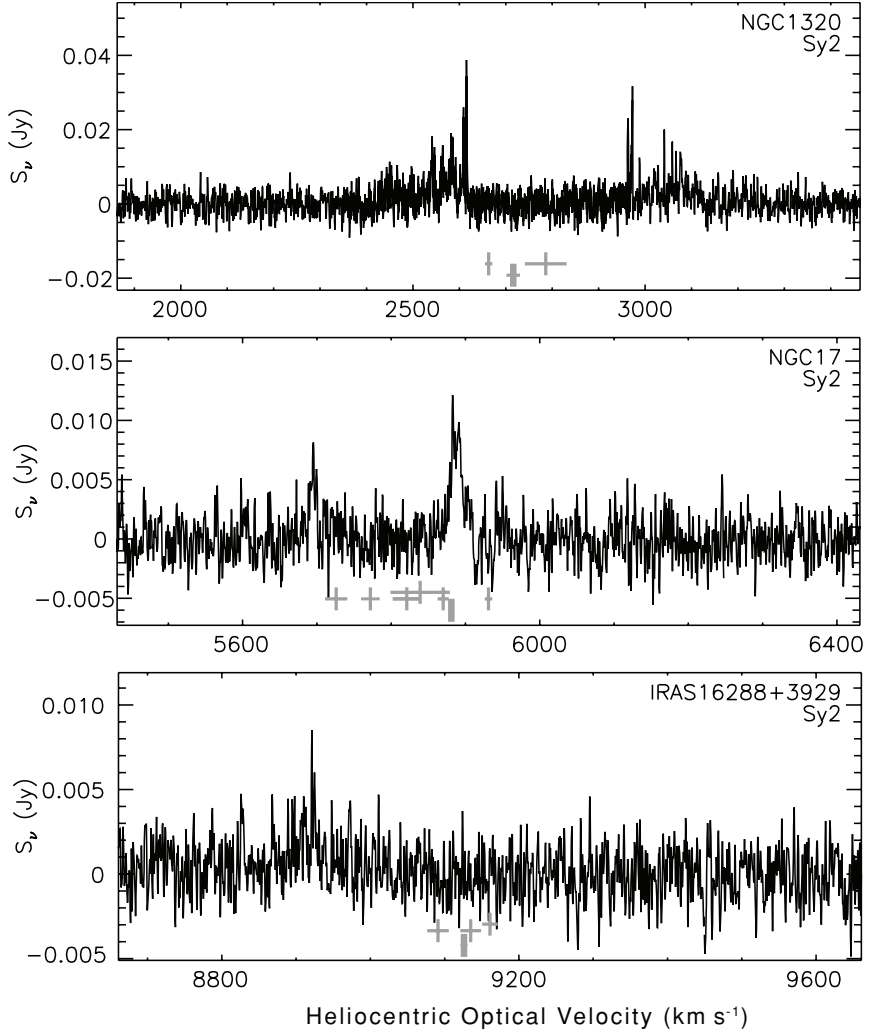

Figure 1. Spectra of newly discovered emission in NGC 1320, NGC 17, and IRAS $16288+3929$. After smoothing, the spectral resolution is $108 \mathrm{kHz}$ $\left(1.5 \mathrm{~km} \mathrm{~s}^{-1}\right)$, for which $1 \sigma$ noise levels are $2.8,1.5$, and $1.3 \mathrm{mJy}$, respectively. Vertical bars indicate systemic velocity estimates. Where there is more than one, the thicker one represents the adopted value, selected based on consideration of error budget. Velocity estimates for NGC 1320 are $2716 \pm 29$ (optical; Huchra et al. 1993), which is adopted here, $2663 \pm 16$ (H I; Davoust \& Contini 2004), and $2786 \pm 90$ (optical; Bottinelli et al. 1992). (We note duplication of NGC designation, and the swapping of MCG identifier and velocity between NGC 1320 and NGC 1321 entries in da Costa et al. 1998.) For NGC 17, the velocities are $5881 \pm 2$ (optical; Rothberg \& Joseph 2006), which is adopted here, $5931 \pm 10$ (H I; Bottinelli et al. 1990), $5772 \pm 25$ (optical; de Vaucouleurs et al. 1991), 5726 \pm 30 (optical; Osterbrock \& Dahari 1983), $5821 \pm 38$ (optical; Huchra et al. 1993), $5821 \pm 44$ (optical; da Costa et al. 1998), $5870 \pm 15$ (optical; Schweizer \& Seitzer 2007), and 5839 \pm 80 (optical; Jones et al. 2005). For IRAS $16288+3929$, optical velocities are $9123 \pm 9$ (emission lines; adopted here) and $9136 \pm 27$ (absorption lines), both from Abazajian et al. (2005), and $9091 \pm 29$ (Zabludoff et al. 1990) and $9161 \pm 21$ (Rines et al. 2002).

galactic Database (NED; Table 1), we have detected three new $\mathrm{H}_{2} \mathrm{O}$ maser sources in Seyfert 2 systems: NGC 1320, NGC 17, ${ }^{3}$ and IRAS 16288+3929 (Figure 1). The three discoveries were subsequently confirmed with the Very Large Array (VLA) of the NRAO using a $6.25 \mathrm{MHz}$ observing bandwidth and 97.7 $\mathrm{kHz}$ channels (NGC 1320, IRAS 16288+3929) or with the GBT on a different day but using the same spectrometer configuration (NGC 17, IRAS 16288+3929). Each of the masers is probably associated with nuclear activity. The NGC 1320 and IRAS $16288+3929$ maser positions measured with the VLA lie $\ll 1 \sigma$ from the optical positions of the nuclei (Table 1), or less than $300 \mathrm{pc}$. The NGC 17 maser is 3-4 times more distant than the farthest $\mathrm{H}_{2} \mathrm{O}$ maser known to be associated with star formation (Arp 244; Darling et al. 2008), yet it is comparably bright.

\footnotetext{
3 Duplicate designation, NGC 34, originates from a suspected observer error recorded in Dreyer (1881) and corrected by Howe (1900). Dual designation does not stem from NGC 17 being a merger with a complicated brightness distribution. However, see discussion of NGC 17/34 in Hopkins et al. (2008).
} 
Table 1

Inclined AGNs Surveyed for $\mathrm{H}_{2} \mathrm{O}$ Maser Emission

\begin{tabular}{|c|c|c|c|c|c|c|c|}
\hline Galaxy $^{\mathrm{a}}$ & Type & $\begin{array}{c}\alpha_{2000}{ }^{\mathrm{b}} \\
\text { (hh mm ss) }\end{array}$ & $\begin{array}{c}\delta_{2000^{\mathrm{b}}} \\
(\mathrm{dd} \mathrm{mm} \mathrm{ss})\end{array}$ & $\begin{array}{c}v_{\text {sys }}{ }^{\mathrm{c}} \\
\left(\mathrm{km} \mathrm{s}^{-1}\right)\end{array}$ & Date & $\begin{array}{l}T_{\text {sys }}{ }^{\mathrm{d}} \\
(\mathrm{K})\end{array}$ & $\begin{array}{c}1 \sigma^{\mathrm{e}} \\
(\mathrm{mJy})\end{array}$ \\
\hline UGC 12915 & LINER & 000141.94 & +232944.5 & 4336 & 2005 Feb 2 & 35 & 5.6 \\
\hline UGC 00050 & LINER & 000640.15 & +260916.2 & 7552 & 2005 Feb 1 & 34 & 6.1 \\
\hline NGC $17 \star$ & Sy2 & 001106.55 & -120626.3 & 5931 & 2006 Apr 18 & 47 & 3.2 \\
\hline UGC 00238 & LINER & 002503.35 & +312042.6 & 6796 & 2005 Feb 1 & 35 & 5.6 \\
\hline M31 & LINER & 004244.32 & +411608.5 & -300 & 2005 Feb 1 & 43 & 7.9 \\
\hline IRAS $01189+2156$ & Sy2 & 012144.27 & +221235.1 & 17478 & 2005 Feb 1 & 35 & 5.3 \\
\hline UM 319 & Sy2 & 012321.18 & -015836.0 & 4835 & 2005 Sep 21 & 56 & 9.2 \\
\hline UGC 00987 & Sy2 & 012531.46 & +320811.4 & 4658 & 2005 Jan 28 & 31 & 5.2 \\
\hline NGC 0660 & LINER & 014301.70 & +133834.0 & 850 & 2005 Feb 2 & 43 & 8.7 \\
\hline UGC 01282 & Sy2 & 014929.87 & +123032.6 & 5221 & 2005 Feb 2 & 38 & 6.5 \\
\hline UGC 01479 & Sy2 & 020019.06 & +242825.3 & 4927 & 2005 Feb 2 & 36 & 5.7 \\
\hline UGC 01757 & Sy2 & 021723.05 & +382449.9 & 5254 & 2005 Jan 28 & 30 & 5.5 \\
\hline UGC 02456 & Sy2 & 025958.59 & +364914.3 & 3605 & 2005 Feb 1 & 42 & 5.9 \\
\hline UGC 02638 & LINER & 031702.21 & +011517.9 & 7123 & 2005 Sep 21 & 54 & 10 \\
\hline NGC $1320 \star$ & Sy2 & 032448.70 & -030232.2 & 2663 & 2005 Sep 30 & 47 & 5.9 \\
\hline MCG -02-09-040 & Sy2 & 032504.94 & -121827.8 & 4495 & 2005 Sep 30 & 48 & 6.0 \\
\hline SBS $0811+584$ & Sy2 & 081601.30 & +582001.0 & 7584 & 2005 Sep 30 & 43 & 5.6 \\
\hline NGC 2683 & Sy2 & 085241.42 & +332513.7 & 411 & 2005 Sep 30 & 44 & 6.5 \\
\hline NGC 3190 & LINER & 101805.77 & +214955.8 & 1271 & 2005 Oct 1 & 46 & 5.9 \\
\hline UGC 05613 & LINER & 102332.50 & +522030.0 & 9620 & 2005 Feb 1 & 34 & 6.0 \\
\hline NGC 3593 & Sy2 & 111437.00 & +124904.0 & 628 & 2005 Oct 1 & 47 & 6.5 \\
\hline NGC 3628 & LINER & 112016.95 & +133520.1 & 843 & 2005 Feb 1 & 52 & 8.1 \\
\hline NGC 3753 & AGN & 113753.90 & +215853.0 & 8727 & 2005 Feb 1 & 40 & 5.5 \\
\hline MCG +03-38-017 & $\mathrm{AGN}$ & 144753.39 & +190437.1 & 12506 & 2005 Feb 1 & 35 & 6.0 \\
\hline IRASF $15033+2617$ & Sy2 & 150527.93 & +260529.3 & 16579 & 2005 Feb 1 & 35 & 5.9 \\
\hline IRASF 15588+6522 & $\mathrm{AGN}$ & 155918.95 & +651357.8 & 8975 & 2005 Feb 1 & 34 & 6.0 \\
\hline IRAS $16288+3929 \star$ & Sy2 & 163032.66 & +392303.2 & 9161 & 2006 Apr 10 & 39 & 2.8 \\
\hline UGC 10593 & Sy2 & 165218.87 & +555419.8 & 8739 & 2005 Sep 21 & 70 & 14 \\
\hline UGC 10695 & LINER & 170505.56 & +430235.1 & 8328 & 2005 Sep 28 & 51 & 6.6 \\
\hline NGC 6503 & LINER & 174927.11 & +700839.6 & 60 & 2005 Sep 28 & 56 & 6.5 \\
\hline $\mathrm{MCG}+11-22-046$ & Sy2 & 182202.20 & +663637.7 & 4393 & 2005 Sep 28 & 57 & 7.0 \\
\hline CGCG 341-006 & Sy2 & 184526.23 & +721101.7 & 13880 & 2005 Feb 1 & 35 & 6.0 \\
\hline NGC 7013 & LINER & 210333.31 & +295349.3 & 779 & 2005 Feb 2 & 38 & 5.9 \\
\hline IC 1417 & Sy2 & 220021.61 & -130849.1 & 5345 & 2005 Sep 30 & 53 & 6.4 \\
\hline NGC 7331 & LINER & 223704.09 & +342456.3 & 816 & 2005 Feb 1 & 42 & 7.2 \\
\hline UGC 12201 & LINER & 224909.55 & +345930.5 & 5055 & 2005 Feb 2 & 34 & 4.9 \\
\hline NGC 7466 & Sy2 & 230203.42 & +270309.5 & 7493 & 2005 Feb 1 & 32 & 5.1 \\
\hline UGC 12318 & Sy2 & 230208.01 & +254013.7 & 9533 & 2005 Feb 1 & 34 & 5.5 \\
\hline NGC 7549 & $\mathrm{AGN}$ & 231517.23 & +190230.1 & 4736 & 2005 Feb 2 & 35 & 5.5 \\
\hline UGC 12519 & Sy2 & 232002.75 & +155710.6 & 4378 & 2005 Feb 1 & 38 & 5.6 \\
\hline
\end{tabular}

\section{Notes.}

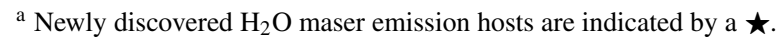

b Optical positions used for GBT pointing. Uncertainties are typically less than $2^{\prime \prime}$. Newly discovered maser emission in NGC 1320 and IRAS $16288+3929$ lies within $1 \sigma$ of the catalog positions for the two nuclei. NGC 1320 : $\alpha_{2000}=03^{\mathrm{h}} 24^{\mathrm{m}} 48^{\mathrm{s}} .70 \pm 0.02 ; \delta_{2000}=-03^{\circ} 02^{\prime} 32^{\prime \prime} \cdot 3 \pm 0.3$ (maser; VLA); $\alpha_{2000}=03^{\mathrm{h}} 24^{\mathrm{m}} 48^{\mathrm{s}} .70 \pm 0^{\mathrm{s}} .08 ; \delta_{2000}=-03^{\circ} 02^{\prime} 32^{\prime \prime} .2 \pm 1^{\prime \prime} .3$ (nucleus; Skrutskie et al. 2006). IRAS16288+3929: $\alpha_{2000}=16^{\mathrm{h}} 30^{\mathrm{m}} 32^{\mathrm{s}} .66 \pm 0.02 ; \delta_{2000}=$ $39^{\circ} 23^{\prime} 03^{\prime \prime} 1 \pm 0 . \prime 3$ (maser; VLA); $\alpha_{2000}=16^{\mathrm{h}} 30^{\mathrm{m}} 32^{\mathrm{s}} .65 \pm 0.02 ; \delta_{2000}=39^{\circ} 23^{\prime} 03^{\prime \prime} .13 \pm 0$. 5 (nucleus; Abazajian et al. 2005).

c Systemic velocity (optical heliocentric) used in tuning the GBT observing bands. Velocities obtained from the NED. When multiple measurements are available, those with the smallest listed uncertainties were chosen, in most cases.

d Average system temperature. Temperatures (and rms noise) during 2005 January and February integrations have been increased by a conservative $30 \%$ factor to correct for apparent miscalibration of the noise source. The correction factor was determined using a receiver temperature of $16.5 \mathrm{~K}$, $6 \mathrm{~K}$ contribution due to spillover and microwave background emission, and archival average forecast ground temperature and $22 \mathrm{GHz}$ opacity.

${ }^{\text {e }}$ rms noise in a $24.4 \mathrm{kHz}$ spectral channel corrected for atmospheric opacity (i.e., referenced to outside the atmosphere), for the dependence of antenna gain on elevation, and for uncertainty in the noise source power level (see note (d); calibration uncertainties are discussed in Section 2).

Spectroscopic classification of sources as disk maser candidates is most suggestive when three line complexes are detected, one close to the systemic velocity and two bracketing it, with similar but opposite velocity offsets. Approximate symmetry in this respect is indicative of a disk, and a minimum offset on the order of $200 \mathrm{~km} \mathrm{~s}^{-1}$ is reasonable to exclude misidentification of emission driven by nonnuclear star formation (Greenhill 2007). However, detailed geometry of the underlying accretion disks, orientation with respect to the line of sight, and sensitivity limitations may result in fewer than three detectable line complexes. In these cases, interpretation of the spectra depends critically on estimates of systemic velocity.

The spectrum of NGC 1320 is relatively symmetric about a mean of $\sim 2790 \mathrm{~km} \mathrm{~s}^{-1}$ (Figure 1), with emission extending outward $\sim 170 \mathrm{~km} \mathrm{~s}^{-1}$ from peaks at 2614 and $2969 \mathrm{~km} \mathrm{~s}^{-1}$. However, the mean velocity is offset $\sim 70 \mathrm{~km} \mathrm{~s}^{-1}$ from the most 
precise optical systemic velocity (Huchra et al. 1993), though agreement appears to be good with the estimate of Bottinelli et al. (1992). The approximate mirror symmetry and breadth of the line complexes are suggestive of $150-180 \mathrm{~km} \mathrm{~s}^{-1}$ rotation velocity, though it would be twice as great if one of the detected line complexes were to correspond to systemic emission. Time monitoring might distinguish between scenarios if a third, blueshifted, variable line complex is detected or if secular drift in velocity is detected in one of the known complexes.

Classification of NGC 17 is somewhat less certain because of a spread in systemic velocity estimates that spans the separation of the two detected line complexes $\sim 300 \mathrm{~km} \mathrm{~s}^{-1}$ (Figure 1). However, we note that the most precise estimate (Rothberg \& Joseph 2006), obtained from dynamical modeling of the galaxy, corresponds well to the peak of the stronger of the two line complexes. We infer from this circumstance that NGC 17 is likely to be a disk maser, with rotation velocity $\sim 300 \mathrm{~km} \mathrm{~s}^{-1}$. As for NGC 1320, monitoring may increase certainty should a blueshifted line complex or secular drift be detected. Nonetheless, because NGC 17 is a merger (e.g., Vorontsov-Vel'Yaminov 1977; Mazzarella \& Boroson 1993; Hunt et al. 1999; Schweizer \& Seitzer 2007), interpretation of the spectrum requires some caution. The velocity offset between the two spectral features is comparable to the stellar velocity dispersion, $\sigma_{\circ}=201 \pm 8 \mathrm{~km} \mathrm{~s}^{-1}$ (Rothberg \& Joseph 2006), and the maser lines may arise in different compact structures within the system.

For IRAS $16288+3929$, classification is problematic because only a single spectral feature is evident $\left(\sim 8920 \mathrm{~km} \mathrm{~s}^{-1}\right)$, and it is displaced $\sim 200 \mathrm{~km} \mathrm{~s}^{-1}$ from available optical systemic velocities (Figure 1). The emission may represent high-velocity blueshifted emission or activity unrelated to any accretion disk. An alternative origin could be jet activity driven by the central engine. We note comparable velocity offsets for known "jet masers" (NGC 1068, NGC 1052, Mrk 348, and M 51; Gallimore et al. 1996, 2001; Claussen et al. 1998; Kameno et al. 2005; Peck et al. 2003; Hagiwara 2007). On the other hand, in these cases, spectral features are characteristically at least $50 \mathrm{~km} \mathrm{~s}^{-1}$ wide, at least an order of magnitude broader than the linewidth for the known maser feature in IRAS $16288+3929$.

NGC 1320 and NGC 17 were targeted but not detected in previous surveys for maser emission. Braatz et al. (1996) and Kondratko et al. (2006b) reported observation of NGC 17 with $1 \sigma$ noise levels of 73 and 16 mJy in 0.66 and $1.3 \mathrm{~km} \mathrm{~s}^{-1}$ spectral channels, respectively. These correspond to signal-tonoise ratios $(\mathrm{S} / \mathrm{N})$ of $\lesssim 1$ for the observed line flux of $11 \mathrm{mJy}$ (peak) over $1.5 \mathrm{~km} \mathrm{~s}^{-1}$. In the case of NGC 1320, these earlier studies obtained $1 \sigma$ noise levels of 61 and $19 \mathrm{mJy}$ in 0.84 and $1.3 \mathrm{~km} \mathrm{~s}^{-1}$ spectral channels, respectively. The $\mathrm{S} / \mathrm{N}$ for the observed line flux of $37 \mathrm{mJy}$, at $1.5 \mathrm{~km} \mathrm{~s}^{-1}$ resolution, would have been less than 2 .

\subsection{Three New Disk Maser Candidates (NGC 449, NGC 2979, and NGC 3735) Among Known Sources}

Through deep integration, we detected new features in the spectra of masers not previously recognized to have highvelocity emission and evidence for origins in accretion disks (Figures 2-4; Table 2). Noise levels were 2-4 mJy $(1 \sigma)$ referenced to outside the atmosphere. We comment on individual systems below. The first three we discuss are the new disk maser candidates.

NGC 449 (Mrk 1) (Figure 2). We detected an emission complex at $\sim 4860 \mathrm{~km} \mathrm{~s}^{-1}$, a $\sim 20$ mJy component at $\sim 4600 \mathrm{~km} \mathrm{~s}^{-1}$,
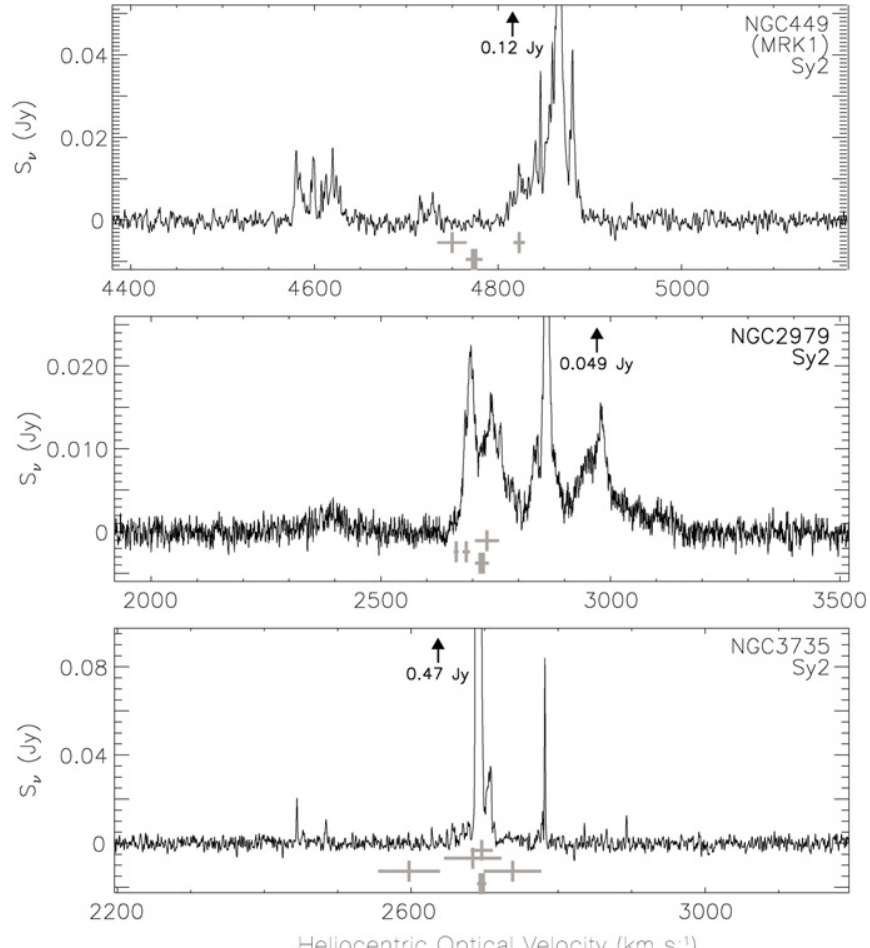

Figure 2. Spectra of known masers in NGC 449 (Mrk 1), NGC 2979, and NGC 3735, obtained with the GBT. After iterative Hanning smoothing, the effective spectral resolution is $1.5 \mathrm{~km} \mathrm{~s}^{-1}$. Vertical bars indicate systemic velocity estimates; where there are more than one, the thicker one represents the adopted value (see Table 2). The vertical scale was expanded to make apparent weak emission. The peak flux densities are noted below the vertical arrows.

and a new weak line complex ( $7 \mathrm{mJy}$ peak in a $1.5 \mathrm{~km} \mathrm{~s}^{-1}$ spectral channel) at $\sim 4720 \mathrm{~km} \mathrm{~s}^{-1}$. The complex at $\sim 4860 \mathrm{~km} \mathrm{~s}^{-1}$ has been present in the spectrum of the source since its discovery (Braatz et al. 1996, 2003), while the component at $\sim 4600 \mathrm{~km} \mathrm{~s}^{-1}$ was marginally detected first by Braatz et al. (2003). The newly detected emission is approximately halfway in velocity between the two previously known spectral complexes. Although the position of the maser emission with respect to the nucleus has not been measured, the spectrum shows the archetypical spectral signature of emission from a highly inclined accretion disk. In particular, considering the uncertainty on the systemic velocity of the galaxy (Figure 2; Table 2), we associate the complex near $4720 \mathrm{~km} \mathrm{~s}^{-1}$ with low-velocity disk emission and infer an orbital velocity (which we take to be the maximum velocity with respect to the systemic velocity) of $\sim 150 \mathrm{~km} \mathrm{~s}^{-1}$. It is notable that the suggested systemic velocity of the galaxy would be blueshifted by a few times $10 \mathrm{~km} \mathrm{~s}^{-1}$ from optical measures and $\sim 100 \mathrm{~km} \mathrm{~s}^{-1}$ from the estimate obtained from $\mathrm{H}$ I data.

NGC 2979 (Figure 2). Emission was newly detected at a level of $\sim 3 \mathrm{mJy}$ at $2390 \mathrm{~km} \mathrm{~s}^{-1}$ (cf. Greenhill et al. 2003a), which is blueshifted by $\sim 300 \mathrm{~km} \mathrm{~s}^{-1}$ from estimates of the systemic velocity. The new spectrum is approximately centered on the optical systemic velocity estimate of $2720 \pm 15 \mathrm{~km} \mathrm{~s}^{-1}$ (Fisher et al. 1995), which is suggestive of emission from a highly inclined disk with $\sim 300 \mathrm{~km} \mathrm{~s}^{-1}$ orbital velocity, though extended line wings may suggest a somewhat more rapid rotation. This interpretation is consistent with the known coincidence of the maser source position and the active nucleus (Greenhill et al. 2003a). Asymmetry in the maser spectrum is pronounced but difficult to explain without imaging data that would enable geometric modeling of the disk. However, we 
note that the archetypal disk maser in NGC 4258 exhibits about a 10 times imbalance in the flux densities of red-and blueshifted emission. In the new spectrum, the velocity range of emission $\gtrsim 10 \mathrm{mJy}$ is consistent with a weak plateau observed in the 6 times lower sensitivity discovery spectrum (Greenhill et al. 2003a).

NGC 3735 (Figure 2). We detected narrow lines at 2444, 2484,2784 , and $2896 \mathrm{~km} \mathrm{~s}^{-1}$ that were not observed in previous spectra (Greenhill et al. 1997b; Braatz et al. 2003). An additional complex of weak lines may be present between 2830 and $2870 \mathrm{~km} \mathrm{~s}^{-1}$. Doppler shifts from systemic velocities derived from $\mathrm{H} \mathrm{I}$ data are $\pm 200-250 \mathrm{~km} \mathrm{~s}^{-1}$ (Staveley-Smith \& Davies 1988). This bracketing of systemic emission and known angular coincidence of the maser and nucleus (Greenhill et al. 1997b) are suggestive of emission from a close-to edge-on accretion disk.

UGC 4203 (Mrk 1210) (Figure 3). We detected line complexes bracketing the systemic velocity, at $3755-3861 \mathrm{~km} \mathrm{~s}^{-1}$ and $4190-4350 \mathrm{~km} \mathrm{~s}^{-1}$ (Figure 3), as well as isolated narrow lines distributed between these, at $4106 \mathrm{~km} \mathrm{~s}^{-1}(\sim 70 \mathrm{mJy})$, $4134 \mathrm{~km} \mathrm{~s}^{-1}$, and $3992 \mathrm{~km} \mathrm{~s}^{-1}$ (5-10 mJy). The peaks of the red- and blueshifted complexes were detected with lower $\mathrm{S} / \mathrm{N}$ previously (Braatz et al. 1996, 2003), though the symmetry of the spectrum around the systemic velocity (Table 2 ) and suggestion of emission from an inclined disk were less pronounced. The inferred orbital velocity is $\sim 300 \mathrm{~km} \mathrm{~s}^{-1}$, depending on the adopted systemic velocity.

Arp 299 (NGC 3690) (Figure 3). The spectrum contains a broad feature with FWHM $\sim 230 \mathrm{~km} \mathrm{~s}^{-1}$ and overlying narrow Doppler components that were not evident in the previous spectra of the source obtained with $4.3 \mathrm{~km} \mathrm{~s}^{-1}$ channels (Henkel et al. 2005). Maser emission is known to arise from three regions in this merging system, the nuclei of NGC 3690 and IC 694 and an internuclear radio continuum "hotspot" (Tarchi et al. 2007). Both nuclear maser sources are $\sim 200 \mathrm{~km} \mathrm{~s}^{-1}$ wide, which is notable because large widths have been associated at least circumstantially with jet activity (see Tarchi et al. 2007), as exemplified in three AGNs where maser and radio continuum emission have been mapped relative to one another interferometrically: NGC 1052, NGC 1068, and Mrk 348 (Claussen et al. 1998; Gallimore et al. 2001; Peck et al. 2003; Kameno et al. 2005, also review by Greenhill 2007). Notable time variability has been observed toward these masers, and position-resolved monitoring of Arp 299 may offer useful diagnostics.

NGC 4293 (Figure 3). The spectrum exhibits emission distributed over $\sim 290 \mathrm{~km} \mathrm{~s}^{-1}$ but that unevenly brackets the most
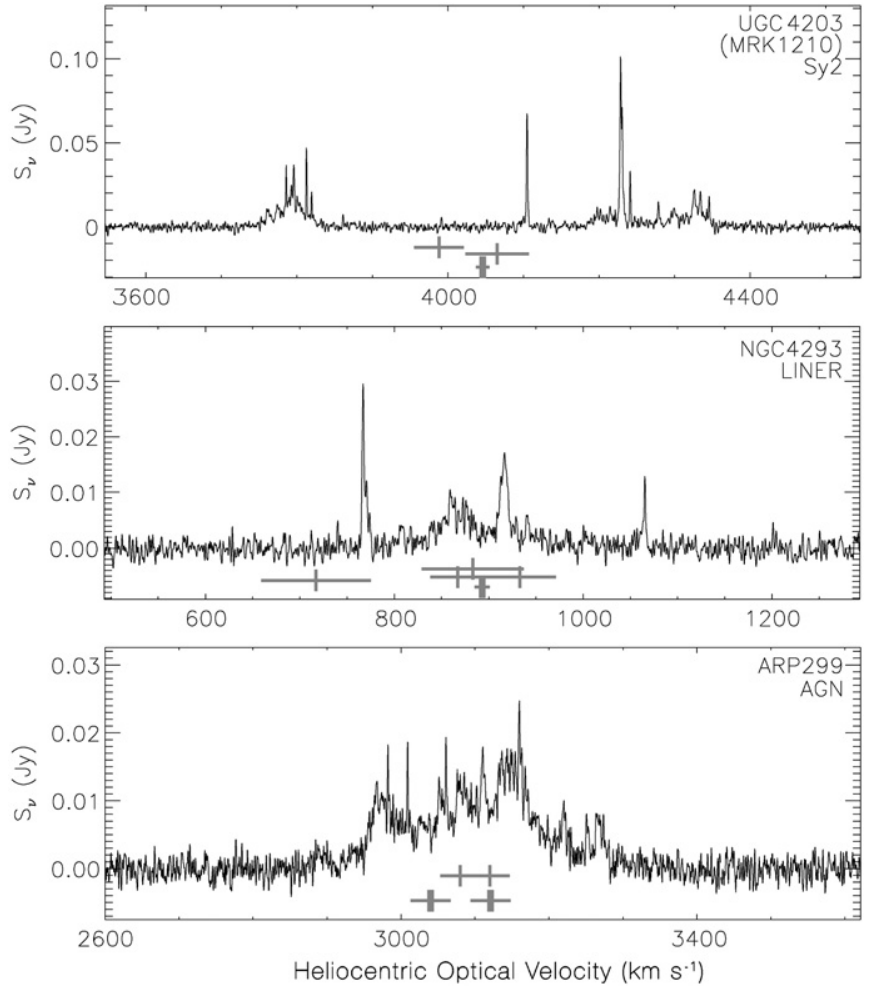

Figure 3. Spectra of known masers in UGC 4203, Arp 299, and NGC 4293, as in Figure 2. For Arp 299, systemic velocities are shown for each nucleus, NGC 3690 and IC 694. See Hibbard \& Yun (1999) for discussion of nomenclature and identification.

precise estimate of systemic velocity (di Nella et al. 1995). An additional emission feature at $\sim 1200 \mathrm{~km} \mathrm{~s}^{-1}$ may be present at the $\sim 3 \sigma$ level. The case for origin in a disk is marginal due to uncertainty in the full velocity range of emission and the degree of symmetry.

NGC 0235A (Figure 4). Dominated by a single spectral feature redshifted by $\sim 300 \mathrm{~km} \mathrm{~s}^{-1}$ from the systemic velocity, interpretation of the spectrum is difficult. Goals of further monitoring should include a search for blueshifted emission.

NGC 2824 (Figure 4). As observed in the case of Arp 299, the NGC 2824 maser exhibits narrow Doppler components overlying a broad component (FWHM $\sim 150 \mathrm{~km} \mathrm{~s}^{-1}$ ). This is consistent with the discovery spectrum (Greenhill et al. 2003a), though the newly recognized zero-intensity width is substantially broadened, $\sim 450 \mathrm{~km} \mathrm{~s}^{-1}$. The maser emission is associated in position with the AGN (Greenhill et al. 2003a), and

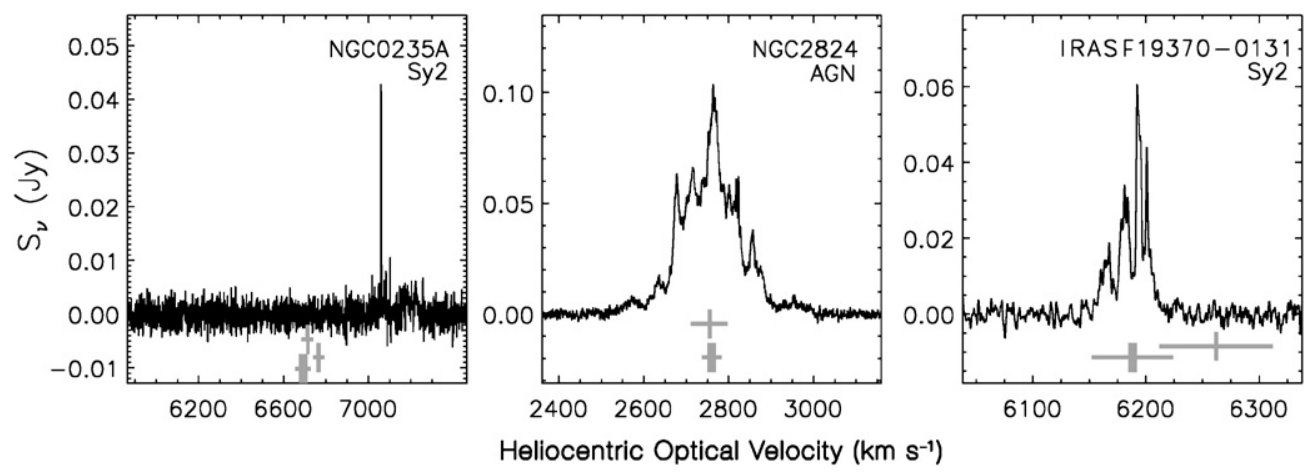

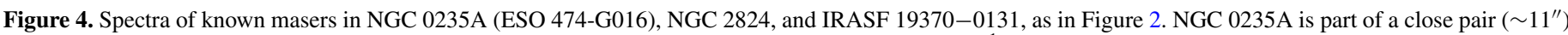

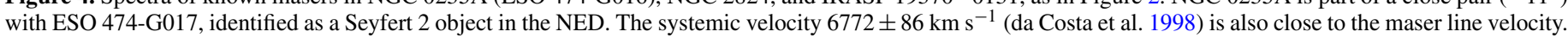


Table 2

Deep Integrations on Known $\mathrm{H}_{2} \mathrm{O}$ Maser Sources

\begin{tabular}{|c|c|c|c|c|c|c|c|}
\hline Galaxy & Type $^{\mathrm{a}}$ & $\begin{array}{c}\alpha_{2000^{\mathrm{b}}} \\
\text { (hh mm ss) }\end{array}$ & $\begin{array}{c}\delta_{2000}{ }^{\mathrm{b}} \\
(\mathrm{dd} \mathrm{mm} \mathrm{ss})\end{array}$ & $\begin{array}{c}v_{\text {sys }}{ }^{\mathrm{c}} \\
\left(\mathrm{km} \mathrm{s}^{-1}\right)\end{array}$ & Date & $\begin{array}{c}T_{\text {sys }} \mathrm{d} \\
(\mathrm{K}) \\
\end{array}$ & $\begin{array}{c}1 \sigma^{\mathrm{e}} \\
(\mathrm{mJy})\end{array}$ \\
\hline NGC 449 (Mrk 1) & Sy2 & 011607.25 & +330522.4 & 4780 & 2006 Apr 19 & 48 & 2.4 \\
\hline NGC 2979 & Sy 2 & 094308.65 & -102300.0 & 2720 & 2006 Feb 9 & 37 & 2.0 \\
\hline NGC 3735 & Sy2 & 113557.30 & +703208.1 & 2696 & 2006 Feb 9 & 37 & 3.9 \\
\hline UGC 4203 (Mrk 1210) & Sy2 & 080405.86 & +050649.8 & 4046 & 2006 Apr 24 & 44 & 3.3 \\
\hline Arp 299 (NGC 3690) & AGN & 112832.20 & +583344.0 & 3121 & 2006 Apr 27 & 46 & 2.9 \\
\hline NGC 4293 & LINER & 122112.82 & +182257.4 & 893 & 2006 Apr 10 & 38 & 2.8 \\
\hline NGC 0235A & Sy2 & 004252.81 & -233227.8 & 6664 & 2006 Feb 1 & 58 & 3.8 \\
\hline NGC 2824 & AGN & 091902.22 & +261612.0 & 2760 & $2006 \operatorname{Jan} 27$ & 35 & 2.5 \\
\hline IRASF 19370-0131 & Sy2 & 193938.91 & -012433.2 & 6188 & 2006 Apr 19 & 48 & 3.7 \\
\hline
\end{tabular}

Notes.

a Activity type from the NED, except for NGC 0235A, which is from Kondratko et al. (2006b).

b GBT pointing positions adopted from Kondratko et al. (2006b) for NGC 0235A, NGC 4293 ( $\sigma=0$ 0"3); from Greenhill et al. (2003a) for NGC 2824, NGC 2979, IRASF 19370-0131 ( $\sigma=0 . \prime 2)$; and from the NED for NGC 449 ( $\sigma=0 . \prime 28)$, UGC $4203(\sigma=0.75)$, Arp $299\left(\mathrm{NGC} 3690, \sigma=10^{\prime \prime}\right)$, NGC 3735 $(\sigma=1 . " 3)$

c Systemic velocity (optical heliocentric) obtained from the NED used in tuning the GBT observing bands. Measurements for individual galaxies follow (in km s ${ }^{-1}$ ). NGC 449 (Mrk 1): $4750 \pm 16$ (optical; Falco et al. 1999), $4774 \pm 9$ (optical; Keel 1996), $4823 \pm 6$ (H I; Bottinelli et al. 1990); NGC 2979: 2664 \pm 5 (H I; Saunders et al. 2000), $2686 \pm 8$ (H I; Paturel et al. 2003), $2720 \pm 15$ (optical; Fisher et al. 1995), $2731 \pm 26$ (optical; Fairall et al. 1992); NGC 3735: $2597 \pm 42$ (optical; Sandage 1978), $2684 \pm 39$ (optical; Falco et al. 1999), $2696 \pm 6$ (H I; Staveley-Smith \& Davies 1988 ), $2696 \pm 15$ (H I; Fisher \& Tully 1981), $2738 \pm 39$ (optical; de Vaucouleurs et al. 1979); UGC 04203 (Mrk 1210): $3988 \pm 33$ (optical; Fisher et al. 1995), $4046 \pm 9$ (H i; Bottinelli et al. 1990), $4065 \pm 42$ (optical; Falco et al. 1999); Arp 299 (NGC 3690): (see Hibbard \& Yun 1999 concerning nomenclature): $3040 \pm 27$ (optical; García-Marín et al. 2006), $3080 \pm 40$ (radio; Zhao et al. 1997); Arp 299 (IC 694): $3120 \pm 50$ (radio; Zhao et al. 1997), $3121 \pm 27$ (optical; García-Marín et al. 2006); NGC 4293: $717 \pm 58$ (optical; de Vaucouleurs et al. 1991), $867 \pm 29$ (optical; J. Huchra 2008, private communication); $883 \pm 54$ (optical; Falco et al. 1999 ), $893 \pm 8$ (optical; di Nella et al. 1995), $933 \pm 38$ (optical; Huchra et al. 1983); NGC 0235A (ESO 474-G016): 6692 \pm 37 (optical; da Costa et al. 1998), 6692 \pm 28 (optical; da Costa et al. 1991), $6712 \pm 28$ (optical; Wegner et al. 2003), $6765 \pm 26$ (optical; Jones et al. 2005); NGC 2824: $2760 \pm 23$ (optical; Huchra et al. 1983), $2755 \pm 44$ (optical; Falco et al. 1999); IRASF 19370-0131: $6188 \pm 36$ (optical; Strauss et al. 1992), 6262 \pm 50 (optical; Jones et al. 2005).

d Average system temperature (not corrected for opacity).

e rms noise in a $24.4 \mathrm{kHz}$ spectral channel corrected for atmospheric opacity (typically from 0.02 to 0.04 ) and for the dependence of antenna gain on elevation.

the spectrum is suggestive of jet activity, but the interpretation is uncertain.

IRASF 19370-0131 (Figure 4). Observed emission is limited to a range $\sim 50 \mathrm{~km} \mathrm{~s}^{-1}$ centered on the systemic velocity estimated by Strauss et al. (1992). Detection of secular drift in feature velocities, as well as emission over a broader range, would be useful goals for future monitoring.

\subsection{New Centripetal Accelerations for Three Disks}

Deep integrations were also used in monitoring three masers reported by Kondratko et al. (2006a), which display among the largest Doppler shifts for high-velocity emission: CG 211, NGC 6264, and VV 340A (Table 3; Figure 5). We detected secular drifts in low-velocity features, which probably reflect centripetal acceleration due to disk rotation (Table 4; Figures 6 and 7). For each source, we used an iterative least-squares technique, adopted from Humphreys et al. (2008), to decompose the time-series spectra into Gaussian components, solving simultaneously for the light curve, time-varying line width, velocity at a reference epoch, and velocity drift of each component. (Each component was assumed to have its own fixed drift rate.) We constrained the widths of most Gaussian components to be $<5 \mathrm{~km} \mathrm{~s}^{-1}$, motivated by empirical observation. A first-order polynomial and a wide $\left(\sim 25 \mathrm{~km} \mathrm{~s}^{-1}\right)$ stationary Gaussian component were used to model the broad low-velocity plateaus in VV 340A and CG 211, respectively. The fitted accelerations were not sensitive to inclusion of the broad components, though reduced $\chi^{2}$ estimates were improved. We obtained drifts of $0.1-$ $1.9 \mathrm{~km} \mathrm{~s}^{-1} \mathrm{yr}^{-1}$ with uncertainties of $0.005-0.09 \mathrm{~km} \mathrm{~s}^{-1} \mathrm{yr}^{-1}$, $1.2-2.1 \mathrm{~km} \mathrm{~s}^{-1} \mathrm{yr}^{-1}$ with uncertainties of $0.1-0.6 \mathrm{~km} \mathrm{~s}^{-1} \mathrm{yr}^{-1}$, and $3.8 \pm 0.1 \mathrm{~km} \mathrm{~s}^{-1} \mathrm{yr}^{-1}$ for CG 211, NGC 6264, and VV 340A, respectively (Table 4 ).
Table 3

Monitoring Log for Secular Drifts

\begin{tabular}{clcc}
\hline \hline Source & \multicolumn{1}{c}{ Epoch } & Time Monitor $^{\mathrm{a}}$ & Mean Spectrum $^{\mathrm{b}}$ \\
\hline CG 211 & 2003 Dec 8 & $\mathrm{x}$ & $\ldots$ \\
& 2005 Jan 15 & $\mathrm{x}$ & $\mathrm{x}$ \\
& 2005 Sep 28 & $\mathrm{x}$ & $\mathrm{x}$ \\
NGC 6264 & 2006 Jan 22 & $\mathrm{x}$ & $\mathrm{x}$ \\
& 2005 Jan 27 & $\ldots$ & $\mathrm{x}$ \\
& 2005 Oct 17 & $\mathrm{x}$ & $\mathrm{x}$ \\
& 2006 Feb 1 & $\mathrm{x}$ & $\mathrm{x}$ \\
VV 340A & 2006 Jun 5 & $\mathrm{x}$ & $\ldots$ \\
& 2003 Dec 13 & $\mathrm{x}$ & $\ldots$ \\
& 2005 Jan 15 & $\mathrm{x}$ & $\mathrm{x}$ \\
& 2005 Jun 7 & $\mathrm{x}$ & $\ldots$ \\
& 2005 Oct 1 & $\mathrm{x}$ & $\mathrm{x}$ \\
& 2006 Feb 1 & $\mathrm{x}$ & $\mathrm{x}$ \\
\hline
\end{tabular}

Notes.

a Epochs over which spectral features were tracked, enabling estimation of accelerations (Figures 6 and 7).

b Epochs used to generate the weighted mean spectrum (Figure 5). For each source, the best three spectra were selected, with particular attention to the flatness of the spectral baseline.

c The CG designation refers to the Case low-dispersion spectroscopic survey (Pesch \& Sanduleak 1983, and later papers by the same authors).

\section{DISCUSSION}

\subsection{Black Hole Masses, Disk Radii, and Their Implications}

Mean measured centripetal acceleration, $a$, combined with mean orbital velocity inferred from single-dish spectra, $v$, can be used to estimate central-engine gravitational masses and accretion disk radii. The mean acceleration among low-velocity features may be expressed as $a_{l}=v_{l}^{2} / R_{l}$, where $v_{l}$ is an 

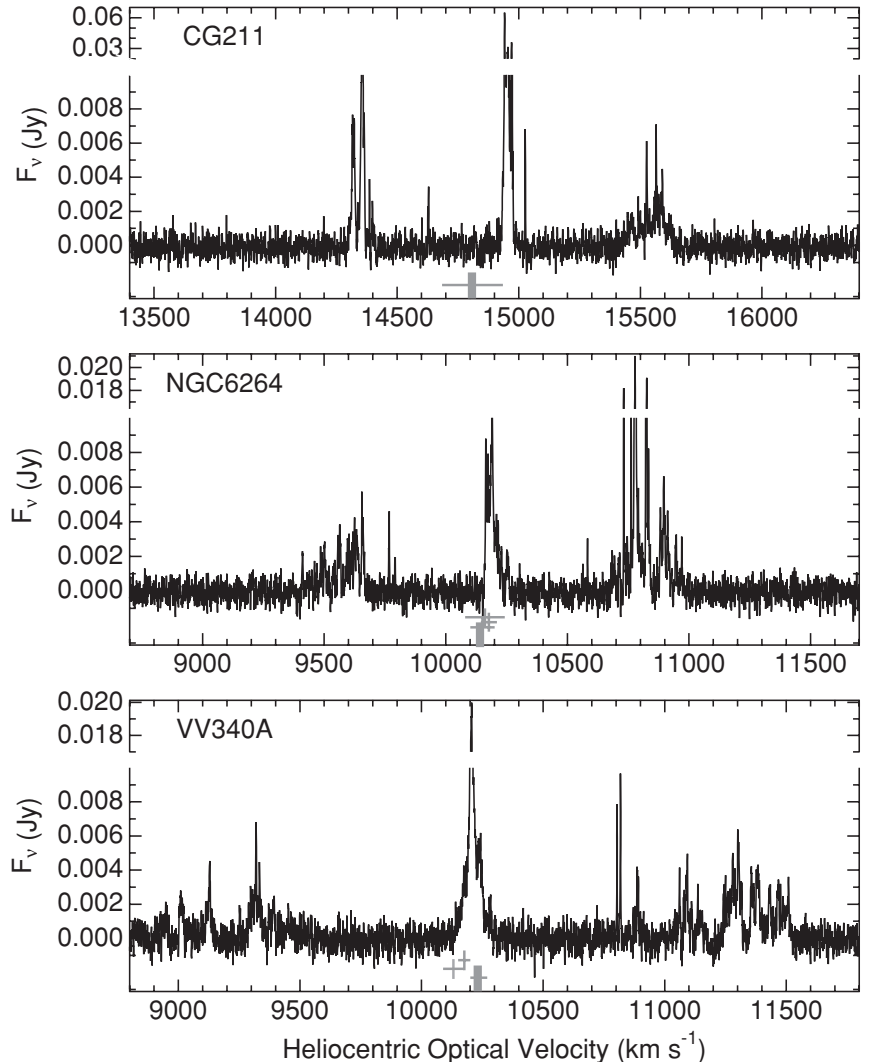

Figure 5. Variance-weighted average spectra for CG 211, NGC 6264, VV 340A, for the epochs listed in Table 3. Spectra have been iteratively Hanning smoothed to a resolution of $1.5 \mathrm{~km} \mathrm{~s}^{-1}$, resulting in $1 \sigma$ noise levels of $0.53,0.42$, and $0.63 \mathrm{mJy}$, respectively. The vertical axes are split to emphasize Doppler components below $10 \mathrm{mJy}$. Vertical bars indicate systemic velocity estimates; where there is more than one, the thicker one represents the adopted value. CG 211: $14810 \pm 120 \mathrm{~km} \mathrm{~s}^{-1}$ (optical; Weistrop \& Downes 1991); NGC 6264: $10141 \pm 10 \mathrm{~km} \mathrm{~s}^{-1}$ (optical; Adelman-McCarthy et al. 2007), $10177 \pm 28 \mathrm{~km} \mathrm{~s}^{-1}$ (optical; Beers et al. 1995), $10161 \pm 76 \mathrm{~km} \mathrm{~s}^{-1}$ (optical; Koranyi \& Geller 2002), $10151 \pm 45 \mathrm{~km} \mathrm{~s}^{-1}$ (optical; Falco et al. 1999); VV 340A: $10234 \pm 29 \mathrm{~km} \mathrm{~s}^{-1}$ (optical; Falco et al. 1999), $10131 \pm 75 \mathrm{~km} \mathrm{~s}^{-1}$ (optical, mean and range; Keel 1996), $10176 \pm 20 \mathrm{~km} \mathrm{~s}^{-1}$ (optical; Karachentsev 1980).

effective mean rotational velocity and $R_{l}$ is the effective mean radius, both for material contributing low-velocity emission. The mean rotational velocity inferred from spectra may be expressed as $v_{h}^{2}=G M / R_{h}$, where $G$ is the gravitational constant, $M$ is black hole mass, and $R_{h}$ is the effect mean radius for material contributing high-velocity emission. For edge-on orientation, we obtain $M=v_{h}^{2} v_{l}^{2}\left(R_{h} / R_{l}\right) / G a_{l}$. Unfortunately, without VLBI, we cannot determine $R_{h}$ and $R_{l}$ independently or, therefore, $v_{h}$ and $v_{l}$ independently. Hence, for the purpose of calculation, we assume that the low- and high-velocity emission arise at the same effective mean radii $\left(R_{h} \sim R_{l} \equiv R\right)$ and that the effective mean rotation velocity of accelerating low-velocity material is the same as the measured mean rotational velocity, $v_{h} \sim v_{l} \equiv v$. We then obtain $M=v^{4} / G a_{l}$ and $R=v^{2} / a$. These values are reported in Table 5. VV 340A may have the largest central mass among known maser galaxies.

We note that the scatter in the velocity drift measurements for CG 211 is substantially larger than the individual formal uncertainties. Since $a=G M / r^{2}$ for low-velocity material and hence $\Delta a / a=-2 \Delta r / r$, the scatter in the measured accelerations of $\Delta a / a \sim 1.0$ might be due to low-velocity emission that populates a wide range of radii within the accretion disk, i.e., $\Delta r / r \sim 0.5$. In our simple model, the
Table 4

Secular Drifts in Low-velocity (Systemic) Emission

\begin{tabular}{|c|c|c|c|c|c|}
\hline Source & Epoch $^{\mathrm{a}}$ & $\chi_{R}^{2 \mathrm{~b}}$ & $\begin{array}{l}\text { Velocity } \\
\left(\mathrm{km} \mathrm{s}^{-1}\right)\end{array}$ & $\begin{array}{c}\text { Drift }^{\mathrm{d}} \\
\left(\mathrm{km} \mathrm{s}^{-1} \mathrm{yr}^{-1}\right) \\
\end{array}$ & Epochs \\
\hline \multirow[t]{10}{*}{$\mathrm{CG} 211^{\mathrm{e}}$} & 2003 Dec 8 & 1.7 & $14940.92 \pm 0.06$ & $0.405 \pm 0.005$ & 4 \\
\hline & & & $14943.2 \pm 0.4$ & $0.90 \pm 0.10$ & \\
\hline & & & $14951.9 \pm 0.1$ & $0.93 \pm 0.03$ & \\
\hline & & & $14954.70 \pm 0.06$ & $0.58 \pm 0.03$ & \\
\hline & & & $14963.9 \pm 0.1$ & $0.85 \pm 0.07$ & \\
\hline & & & $14967.7 \pm 0.1$ & $1.55 \pm 0.04$ & \\
\hline & & & $14969.9 \pm 0.1$ & $1.88 \pm 0.07$ & \\
\hline & & & $14976.0 \pm 0.2$ & $0.11 \pm 0.05$ & \\
\hline & & & $14949.2 \pm 0.9$ & $1.45 \pm 0.09$ & \\
\hline & 2003 Dec 8 & 1.0 & $15025.5 \pm 0.1$ & $\begin{array}{c}0.65 \pm 0.04 \\
(\text { avg. }=0.9)^{\mathrm{f}}\end{array}$ & \\
\hline \multirow[t]{9}{*}{ NGC 6264} & 2005 Oct 17 & 1.2 & $10164.0 \pm 0.2$ & $1.9 \pm 0.2$ & 3 \\
\hline & & & $10164.4 \pm 0.3$ & $1.7 \pm 0.5$ & \\
\hline & & & $10168.2 \pm 0.3$ & $2.1 \pm 0.3$ & \\
\hline & & & $10174.7 \pm 0.3$ & $1.7 \pm 0.6$ & \\
\hline & & & $10181.9 \pm 0.3$ & $2.1 \pm 0.6$ & \\
\hline & & & $10184.7 \pm 0.4$ & $2.0 \pm 0.4$ & \\
\hline & & & $10186.0 \pm 0.1$ & $2.1 \pm 0.1$ & \\
\hline & & & $10188.7 \pm 0.2$ & $2.0 \pm 0.2$ & \\
\hline & & & $10191.0 \pm 0.2$ & $\begin{array}{c}1.2 \pm 0.4 \\
(\text { avg. }=2)^{\mathrm{f}}\end{array}$ & \\
\hline VV $340 A^{e}$ & 2003 Dec 13 & 1.4 & $10199.6 \pm 0.1$ & $3.8 \pm 0.1$ & 5 \\
\hline
\end{tabular}

Notes.

a Reference epoch for component velocities.

b Reduced chi-squared for the least-squares decomposition of line profiles into Gaussian components, in which reference-epoch velocities, secular drifts, time-varying widths, and light curves are simultaneously fit for each Gaussian component using a time-series of spectra. Only components with $\mathrm{S} / \mathrm{N}>3$ for two or more epochs are reported.

${ }^{c}$ Reference epoch for fitted velocity.

d Velocity drift of a Gaussian component.

e Low-velocity plateaus were modeled by an FWHM $\sim 25 \mathrm{~km} \mathrm{~s}^{-1}$ stationary Gaussian component for CG 211 and a first-order polynomial for VV 340A. These components are not reported here.

${ }^{\mathrm{f}}$ Unweighted arithmetic average for use in the calculation of entries in Table 5 (see discussion in Section 4.1).

radial spread among high-velocity material might be expected to be $\Delta v / v=0.5 \Delta r / r \sim 0.25$. The observed value of $\Delta v / v$ in the high-velocity components is about 0.3 (see Figure 5). This suggests that the radial spread is about the same for both high- and low-velocity masers. For NGC 6264, $\Delta a / a \sim 0.4$, so $\Delta r / r \sim 0.2$, and $\Delta v / v$ for the high-velocity masers is expected to be about 0.1 . However, inspection of the spectrum (Figure 5) suggests that $\Delta v / v \sim 0.5$. Hence, the high-velocity masers probably occupy a larger range of radii than the low-velocity masers. Note that the case of NGC 6264 may be similar to that of NGC 4258, where the radial range of the high-velocity features is also greater than that of the low-velocity features (Herrnstein et al. 2005).

In each case where we report centripetal acceleration, it has been positive, i.e., spectral components drift toward larger velocities. This is consistent with the emission being from material on the near side of the disks. Material behind a dynamical center, which would exhibit negative centripetal accelerations, has not been detected toward any known disk maser or disk maser candidate. Although the relatively low S/N achieved for CG 211, NGC 6264, and VV 340A would make recognition of "back-side" emission relatively difficult, we can exclude the possibility that flux densities are equivalent to the 


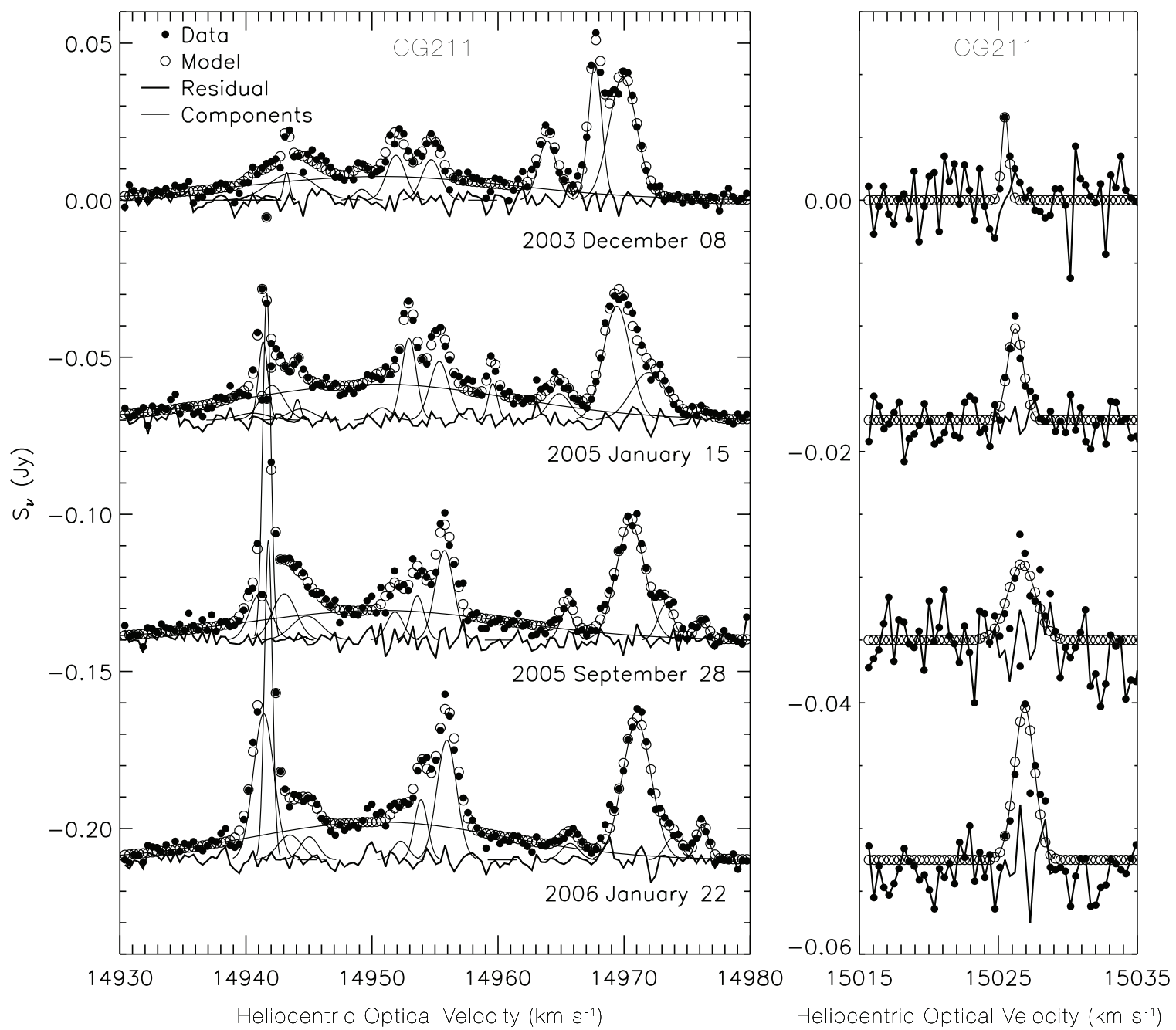

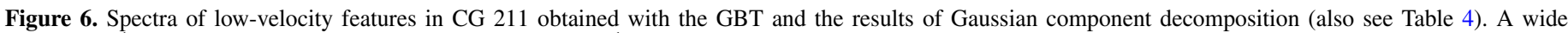
$\left(\sim 25 \mathrm{~km} \mathrm{~s}^{-1}\right)$ stationary Gaussian component at $\sim 14951 \mathrm{~km} \mathrm{~s}^{-1}$ represents a broad low-velocity plateau present at each epoch.

front-side emission. It is difficult to know whether the apparent absence of back-side emission may be due to a maser radiation propagation effect (e.g., competitive gain diminishes inwarddirected beams) or differential absorption (e.g., inward-directed beams pass through regions of enhanced scattering or absorption in the vicinity of a central engine; inward-directed beams cross the full breadth of a disk and are attenuated by high emissionmeasure material at large disk scale heights, as in Herrnstein et al. 1996).

The three masers in Table 5 are priority targets for interferometric programs that are intended to estimate geometric distances (e.g., Herrnstein et al. 1999) and (in combination with distances for other maser targets) a combined Hubble constant, $H_{0}$. For a sample of $N$ anchor galaxies, uncertainty in $H_{0}$ scales as $\sim N^{-0.5}$ (for uniform uncertainties) because errors for individual distance measurements are largely uncorrelated; they depend principally on the distribution of Doppler components across the disk face and details of disk geometries, which differ from galaxy to galaxy.

To provide substantive independent constraint on additional cosmological parameters, such as a time-variable equation of state (EOS) for dark energy and curvature, estimates of $H_{0}$ need to achieve on the order of $1 \%$ uncertainty, matching the growing accuracy of other measurements, e.g., cosmic microwave background (CMB) fluctuations and baryon acoustic oscillations (Hu 2005; Ichikawa \& Takahashi 2008; Olling 2007; Greenhill et al. 2009, and references therein). In combination with CMB data alone, a $1 \%$ estimate of $H_{0}$ would enable a $5 \%$ constraint on a presumed-constant EOS, for a flat universe (Figure 14, Riess et al. 2009). A sample on the order of 100 disk masers would be required to achieve $1 \%$ accuracy in $H_{0}$, if each distance is accurate to $10 \%$. At present, this is at least an order of magnitude beyond the number of disk masers apparently suitable for distance measurement. However, a three-year survey of 3000 galaxies at the GBT, achieving rms noise $\sim 3 \mathrm{mJy}$ over $1 \mathrm{~km} \mathrm{~s}^{-1}$ (4 sources per hour for $\sim 750^{\mathrm{h}}$ ), would result in the detection of 150 masers, assuming a conservative detection rate of one-half of that reported by Kondratko et al. (2006a) for AGNs at $10,000-15,000 \mathrm{~km} \mathrm{~s}^{-1}$. For a disk maser fraction of onethird, and a further one-quarter that appear suitable for distance measurement (as suggested in the currently known population), it would be possible to assemble a sample on the order of 25 objects. For $10 \%$ distance uncertainty per maser, this would enable estimation of $H_{0}$ with $2 \%$ accuracy in the near term. Prospects are improved by better individual distances as may be achievable with the VSOP-2 space-VLBI mission and larger target samples made accessible by the Square Kilometer Array (Greenhill 2004; Morganti et al. 2004).

However, peculiar radial motions of galaxies increase scatter in the Hubble relation and introduce partial correlation among 

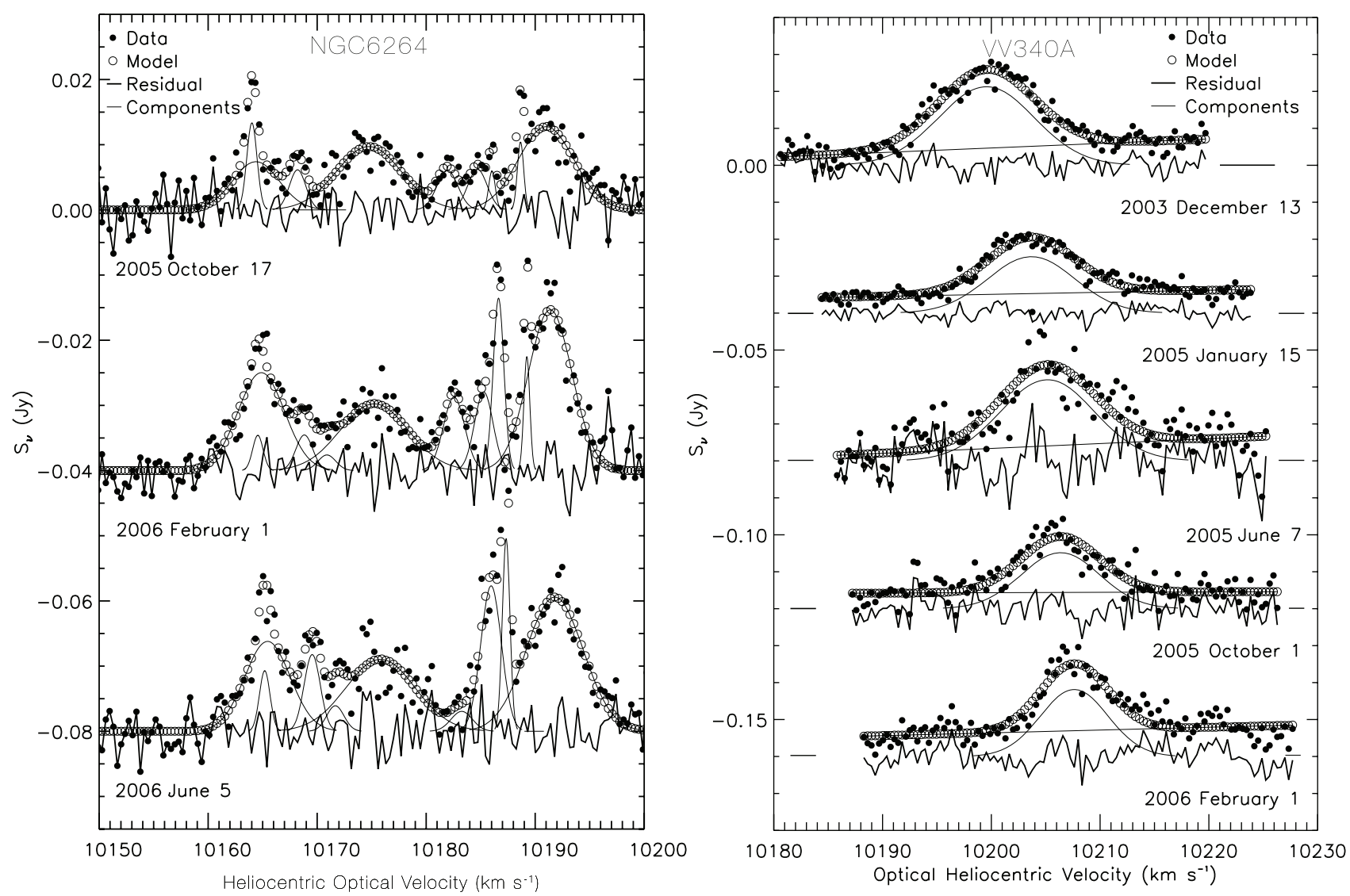

Figure 7. Spectra of low-velocity features in NGC 6264 (left) and VV 340A (right) obtained with the GBT and the results of Gaussian component decomposition (see also Table 4). In the case of VV 340A, horizontal lines to the left and right of the spectra mark the zero level, which is distinguished from the low-level broad emission component.

Table 5

Estimate of Enclosed Mass and Disk Radii

\begin{tabular}{lcc}
\hline \hline Galaxy & $\begin{array}{c}\text { Radius }^{\mathrm{a}} \\
(\mathrm{pc})\end{array}$ & $\begin{array}{c}\text { Mass }^{\mathrm{a}} \\
\left(10^{7} M_{\odot}\right)\end{array}$ \\
\hline CG 211 & 0.4 & 3 \\
NGC 6264 & 0.2 & 2 \\
VV 340A & 0.3 & 7 \\
\hline
\end{tabular}

Note. Because of the uncertainties due to the lack of VLBI data as discussed in Section 4.1, the accuracies of the masses and radii may be as large as half an order of magnitude.

recessional velocities. The estimate of the Hubble constant from any particular galaxy measurement is $D / v$. The sample size needed to achieve a given total uncertainty grows as the quadrature sum of $\Delta D / D$ and fractional peculiar motion, $v_{\text {pec }} / v_{\text {sys }}$, so that the uncertainty in a sample of $N$ galaxies is $\Delta H_{0} / H_{0} \sim\left[\left(v_{\text {pec }} / v_{\text {sys }}\right)^{2}+(\Delta D / D)^{2}\right]^{1 / 2} N^{-1 / 2}$. If $\Delta D / D=0.1$, and we require that the uncertainty in peculiar motion raises the error by less than $10 \%$, then $v_{\text {pec }} / v_{\text {sys }}<0.05$. Outside of clusters, $v_{\text {pec }} \lesssim 500 \mathrm{~km} \mathrm{~s}^{-1}$ is typical (Springob et al. 2007). We take this to be an rms value and infer that $v_{\mathrm{sys}} \gtrsim 10^{4} \mathrm{~km} \mathrm{~s}^{-1}$ is needed. For maser hosts inside rich clusters, barycenter velocities can be estimated with uncertainty $<200 \mathrm{~km} \mathrm{~s}^{-1}$ (e.g., Crook et al. 2007) and used in place of individual galaxy recessional velocities, though cluster peculiar motions would still deserve attention.

It would be desirable to target relatively nearby galaxies that are in "quiet" portions of the Hubble flow, to reduce statistical and nonstatistical errors (e.g., UGC 3789; Masters 2005; Reid et al. 2009). However, a broad distribution of sample members in velocity and angle on the sky is needed. Subtraction of cosmic flow models to at least partially correct for $v_{\text {pec }}$ reduces the lower limit on acceptable $v_{\text {sys }}$ and admits galaxies in not so quiet regions. Using Tully-Fisher distances to estimate peculiar velocities and construct a multi-attractor model inside $6000 \mathrm{~km} \mathrm{~s}^{-1}$, Masters (2005) obtained a $160 \pm 20 \mathrm{~km} \mathrm{~s}^{-1}$ rms residual. Erdoğdu et al. (2006) reported a somewhat less direct error estimate, a scatter of $100-150 \mathrm{~km} \mathrm{~s}^{-1}$ around a flow model derived from galaxy recessional velocities (as opposed to peculiar velocities), up to $\sim 16,000 \mathrm{~km} \mathrm{~s}^{-1}$. Adopting a characteristic $160 \mathrm{~km} \mathrm{~s}^{-1}$ uncertainty, the lower limit in $v_{\text {sys }}$ is $3200 \mathrm{~km} \mathrm{~s}^{-1}$, for maser distances good to $10 \%$ and the nominal limit of $\sim 10 \%$ contribution by peculiar motions to the error budget. The technique works for maser hosts away from the Great Attractor and the galactic plane $\left(|b|>20^{\circ}\right.$; Masters 2005), where flow models are not well constrained.

\subsection{Accretion Disk and Host Galaxy Misalignments}

In studies of the relative orientation of AGN central engines and host galaxies, radio jet position angles at parsec and kiloparsec scales are used to infer the orientations of the accretion disks that launch them. Ionization cone position angles are used to infer the orientations of larger scale, dusty, geometrically thick structures at larger radii. Comparisons of position angles suggest that accretion disks are not aligned with galactic spiral disks (Ulvestad \& Wilson 1984; Nagar \& Wilson 1999; Kinney et al. 2000; Schmitt et al. 2003; Middelberg et al. 
2004). At the same time, the accretion disks appear reasonably well aligned with the structures that collimate ionization cones (Schmitt et al. 2003), though the sample is small.

Because interferometric mapping has demonstrated that disk maser emission marks molecular structures that are oriented close to edge-on, the emission provides a relatively precise indicator of orientation, via inclination rather than position angle, as is otherwise the case principally for radio jets and ionization cones. Thus, estimation of stellar disk inclination in spiral galaxies that host disk masers enables immediate estimation of (mis)alignment between parsec-scale and kiloparsecscale structures. The approach complements other techniques, in terms of orientation angle (position angle versus inclination) and of radius, where the range of radii for maser emission lies outside the zone where accretion disks act to collimate radio jets. Perhaps the fullest case studies would be possible for galaxies with observable radio jets, disk maser emission, well-resolved ionization cones, accurate $B$-band isophotal radii (for detection of spiral arms), and a reasonably firm morphological type. Among nearby galaxies, inclinations could be obtained from detailed modeling of spectroscopic data for well-resolved stellar disks and circumnuclear molecular rings (e.g., Curran 2000).

A suitable sample for study may be compiled directly from lists of known disk maser candidate hosts that are spirals. The spectroscopic identification of masers that trace underlying disk structure is sufficient. Galactic inclinations, to first order, may be inferred directly from axial ratios, $i=\cos ^{-1}(b / a)$, though detailed dynamical modeling would be preferred to obtain the most accurate values.

Assuming the spectroscopic identifications are accurate, then there is no apparent preponderance of edge-on galactic disks among disk maser candidate hosts. For inclinations $<70^{\circ}-80^{\circ}$, the distribution of orientations for the disk maser candidate matches what would be anticipated for random galaxy orientations (Figure 8). To make the comparison, we assembled a sample of axial ratios for published disk maser candidates, defined with respect to surface brightness of $B=25.0 \mathrm{per} \mathrm{s}^{-2}$, for S0 and later systems (Table 6). Axial ratios were taken from the Third Reference Catalogue of Bright Galaxies (de Vaucouleurs et al. 1991).

The apparent undercount among high-inclination systems in Figure 8 is probably due to overestimation of disk minor axes due to galactic bulges, which is anticipated to be greatest for nearly edge-on massive systems (i.e., morphologies from S0 to Sa). Fouque et al. (1990) described an empirical correction that is dependent on galaxy morphology, quantified in the $T$ parameter (e.g., de Vaucouleurs et al. 1991). Determination of morphologies is strongly dependent on image quality and may be affected by subjective bias (e.g., see methodology discussion of Fukugita et al. 2007). Corrected inclinations are particularly sensitive to uncertainty in $T$ for highly inclined systems, where forbidden values of $\cos i$ may result. We estimate corrected inclinations using cataloged morphological types (de Vaucouleurs et al. 1991; Malkan et al. 1998; Lauberts \& Valentjin 1989). For galaxies with forbidden values of $\cos i$ (NGC 1386, NGC 4388, NGC 3735), we increment $T$ by 0.6 , which is approximately the cataloged uncertainty for these systems. We obtain inclinations that are within a few degrees of $90^{\circ}$. (In light of the chosen coarse binning, the precise values are not critical.) As a result, agreement with what is expected for random galactic disk orientations is obtained over the whole range (Figure 8). For an intensively studied sample that includes well-characterized inclination uncertainties for
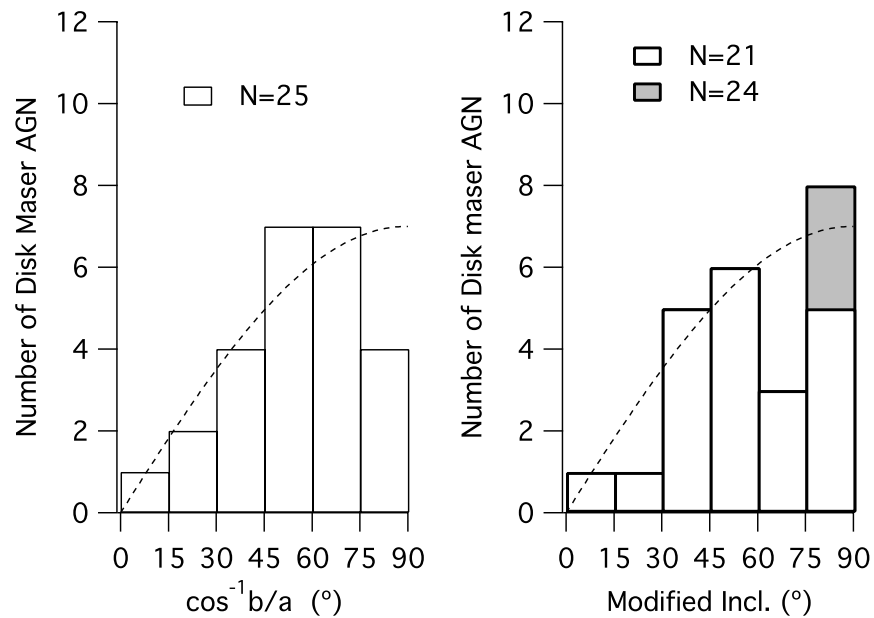

Figure 8. Histograms of inclinations for disk maser candidate host galaxies (Table 6) demonstrating misalignment with the edge-on reference planes defined by disk maser candidates on sub-parsec scales. (Left) Inclination computed from cataloged axial ratios at $B=25$ mag arcsecond $^{-2}$ (de Vaucouleurs et al. 1991), for published disk maser candidates that lie in spiral hosts. The dashed line represents the expectation for a sample of randomly oriented galaxies, scaled to seven objects in the highest bin. (Right) Inclination computed with correction for distortion of isophotes due to galactic bulges (Fouque et al. 1990), using estimates of morphological type $T$ (de Vaucouleurs et al. 1991; Malkan et al. 1998; Lauberts \& Valentjin 1989). Shaded bars represent three galaxies with inclinations very close to $90^{\circ}$ and for which a $\sim 1 \sigma$ or +0.6 adjustment to $T$ (e.g., $\mathrm{Sa} \rightarrow \mathrm{Sab}$ ) was required to obtain allowable values of $\cos i$.

each object, formal statistical comparison would be possible using a Monte Carlo analysis.

No correlation of misalignment with morphological type or mass is apparent. Disk maser candidate hosts span a range of almost 2 orders of magnitude in black hole mass and a broad range of apparent morphological type, from S0 $(T=-2)$ to Sc $(T \leqslant 5)$, excluding the merger system NGC 17 . The median type is 2.7 , with $1<T<4$ for the middle quartiles (Table 6). Masses are inferred from VLBI or acceleration measurements: NGC 4258 (e.g., Miyoshi et al. 1995; Herrnstein et al. 2005), NGC 1068 (Greenhill \& Gwinn 1997), Circinus (Greenhill et al. 2003a), NGC 3079 (Yamauchi et al. 2004; Kondratko et al. 2005), UGC 3789 (Reid et al. 2009), NGC 6323 (Braatz et al. 2007), IC 2560 (Ishihara et al. 2001), Mrk 1419 (Henkel et al. 2002), and CG 211, NGC 6264, and VV 340A (this work).

Comparison of galactic plane misalignments inferred for disk masers and for accretion disks at smaller radii thus far is statistical. Direct comparison would require disk masers that have been mapped with VLBI, but that sample is comparatively small. In several examples, the position angles for jets (or lobes) and ionization cones are well aligned with disk maser rotation axes, projected on the sky: NGC 3393 (Kondratko et al. 2008), NGC 4258 (Cecil et al. 2000), and NGC 4945 (Greenhill et al. 1997c). However, misalignment between a jet and disk maser has also been observed in the case of NGC 3079 (Kondratko et al. 2005), and skewing of ionization cone orientation by shadowing from a warped disk has been observed in Circinus (Greenhill et al. 2003b), both at radii $\lesssim 1 \mathrm{pc}$. Misalignment on the order of $30^{\circ}$ is also observed in NGC 1068, between the position angle of the disk maser and the major axis of ionized material just inside the inner radius for molecular emission (Gallimore et al. 2004). If these examples are representative, counterparts may contribute to scatter in the alignment of jets and ionization cones noted by Schmitt et al. (2003) but ascribed to S/N and projection effects. Warping as observed among disk masers and 
Table 6

Inclinations of Galaxies that have Candidate or Confirmed Disk Masers

\begin{tabular}{|c|c|c|c|c|c|}
\hline \multirow[t]{2}{*}{ Source $^{\mathrm{a}}$} & \multirow[t]{2}{*}{$T^{\mathrm{b}}$} & \multirow{2}{*}{$\begin{array}{c}\cos ^{-1}(b / a)^{\mathrm{c}} \\
\left(^{\circ}\right)\end{array}$} & \multirow{2}{*}{$\begin{array}{c}i_{\text {mod }}{ }^{\mathrm{d}} \\
\left(^{\circ}\right)\end{array}$} & \multicolumn{2}{|c|}{$\begin{array}{c}\text { Evidence for Disk } \\
\text { Maser }^{\mathrm{e}}\end{array}$} \\
\hline & & & & $\begin{array}{l}\text { Spectrum } \\
\text { Ref. }\end{array}$ & $\begin{array}{l}\text { Map } \\
\text { Ref. }\end{array}$ \\
\hline NGC $4945^{f}$ & 6 & 79 & 86 & 1 & 1 \\
\hline Circinus $^{\mathrm{f}}$ & 3 & 64 & 69 & 2,3 & 16 \\
\hline NGC $4258^{f}$ & 4 & 67 & 71 & 4 & 17 \\
\hline NGC $1386^{f}$ & -0.6 & 68 & (87) & 5 & 18 \\
\hline NGC $1068^{f}$ & 3 & 32 & 33 & 6 & 19 \\
\hline NGC $3079^{f}$ & 7 & 80 & 85 & 7 & 20 \\
\hline NGC 4388 & 3 & 77 & (87) & 8 & $\ldots$ \\
\hline NGC 3735 & 5 & 78 & (86) & 9 & $\ldots$ \\
\hline NGC 1320 & 1 & 70 & 85 & 9 & $\ldots$ \\
\hline NGC 5728 & 1 & 54 & 60 & 8 & $\ldots$ \\
\hline IC $2560^{f}$ & 3.3 & 51 & 53 & 5 & 21 \\
\hline NGC5்793 & 3 & 70 & 77 & 10 & $\ldots$ \\
\hline NGC $3393^{f}$ & 1 & 24 & 26 & 11 & 22 \\
\hline NGC 591 & 0 & 34 & 37 & 8 & $\ldots$ \\
\hline Mrk 1419 & 1 & 46 & 50 & 12 & $\ldots$ \\
\hline NGC 6926 & 4 & 46 & 48 & 13 & $\ldots$ \\
\hline NGC 5495 & 4.6 & 37 & 38 & 11 & $\ldots$ \\
\hline NGC $6323^{f}$ & 2 & 71 & 81 & 8 & 23 \\
\hline NGC 2979 & 1 & 51 & 55 & 9 & $\ldots$ \\
\hline UGC $3789^{f}$ & 2 & 29 & 31 & 14 & 24 \\
\hline UGC 3193 & 2.3 & 68 & 74 & 14 & $\ldots$ \\
\hline UGC 4203 & 1 & 0 & 0 & 9 & $\ldots$ \\
\hline NGC 449 & 5 & 52 & 54 & 9 & $\ldots$ \\
\hline ESO 269-G012 & -2 & 39 & 41 & 13 & $\ldots$ \\
\hline NGC 6264 & $\ldots$ & 51 & $\ldots$ & 15 & $\ldots$ \\
\hline
\end{tabular}

\section{Notes.}

${ }^{a}$ Published disk maser candidates, in order of increasing recessional velocity. Missing isophotal sizes or merger activity exclude IRASF 22265-1826; CG 211, VV 340A, 3C 403, NGC 17.

b Morphological classification.

${ }^{c}$ Inclination from axial ratio $b / a$.

${ }^{\mathrm{d}}$ Modified inclination (Fouque et al. 1990). Numbers in parentheses are obtained by adding $1 \sigma$ to $T$ to obtain real inclinations.

e References for identification of disk maser candidates via spectroscopy: (1) Greenhill et al. 1997c; (2) Nakai et al. 1995; (3) Greenhill et al. 1997a; (4) Nakai et al. 1993; (5) Braatz et al. 1996; (6) Claussen \& Lo 1986; (7) Hagiwara et al. 2002; (8) Braatz et al. 2004; (9) this work; (10) Hagiwara et al. 1997; (11) Kondratko et al. 2006b; (12) Henkel et al. 2002; (13) Greenhill et al. 2003a; (14) Braatz \& Gugliucci 2008; (15) Kondratko et al. 2006a. References for confirmation of disk maser identification via VLBI study: (16) Greenhill et al. 2003b; (17) Argon et al. 2007; (18) Braatz et al. 1997a; (19) Greenhill \& Gwinn 1997; (20) Kondratko et al. 2005; (21) Ishihara et al. 2001; (22) Kondratko et al. 2008; (23) Braatz et al. 2007; (24) Reid et al. 2009.

${ }^{\mathrm{f}}$ Disk maser status confirmed by VLBI observations.

larger nuclear structures (e.g., Schinnerer et al. 2000) may also be responsible, where fueling of central engines does not depend on conveyance of material along galactic planes to ever smaller radii and vertical heights until it is concentrated into a coplanar accretion disk.

\section{CONCLUSIONS}

In a survey with the GBT of 40 inclined AGNs, we have detected three new water maser sources (NGC 1320, NGC 17, and IRAS 16288-3929; Figure 1). We classify two of these (NGC 1320 and NGC 17) as disk maser candidates based on their spectra. Three more cases of disk maser candidates were discovered through deep integrations toward known masers (NGC 449, NGC 2979, and NGC 3735; Figure 2). One pre- viously marginal case was confirmed (UGC 4203; Figure 3). Inferred rotation speeds are $\sim 100-300 \mathrm{~km} \mathrm{~s}^{-1}$. At present, the details of interpretation are sensitive to estimates of systemic velocity at optical wavelengths and $21 \mathrm{~cm}$ (the $\mathrm{H}$ I line). These show significant scatter for some galaxies, and in general, comprehensive study is mandatory for each new maser detection to resolve uncertainties in classification. Interferometric followup of discoveries reported here will enable confirmation studies of disk geometry (e.g., radii, warping) and estimation of central engine mass, at least for the sources with large numbers of maser Doppler components. In general, systematic velocities that result from disk models may provide the best estimates of systemic velocity, and these might usefully be compared to the results of pointed optical and molecular line studies that include dynamical modeling.

For three additional maser sources exhibiting high-velocity maser emission (CG 211, NGC 6264, VV 340A), we report secular drift in multiple low-velocity features-a probable manifestation of centripetal acceleration due to disk rotation. Combined with rotation velocities inferred from spectra, these centripetal accelerations suggest central engine masses of a few times $10^{7} M_{\odot}$ and mean disk radii of a few times $10^{-1} \mathrm{pc}$. Measurable accelerations of $\sim 1-3 \mathrm{~km} \mathrm{~s}^{-1} \mathrm{yr}^{-1}$ and large recessional velocities $\left(>10^{4} \mathrm{~km} \mathrm{~s}^{-1}\right)$ make these three sources particularly attractive for interferometric study in pursuit of robust estimates of geometric distances and $H_{0}$.

As beacons of highly inclined structures in AGNs, at radii of $\sim 0.1-1 \mathrm{pc}$, disk masers also highlight those galactic nuclei in which we can define a reference plane with high accuracy and study (mis)alignment with surrounding kiloparsec-scale stellar disks. We find no preponderance of edge-on galactic disks among published disk maser candidate hosts. The comparison is in agreement with studies that infer orientations of accretion disks and geometrically thick tori from radio jets and ionization cones, though the maser data refer to a unique range of radii and probe inclination rather than position angle on the sky.

Our studies provide more candidates for the quest to measure $H_{0}$ with disk masers. The best available estimate of $H_{0}$ is uncertain by $5 \%$ and achieved with a composite analysis of Cepheid and supernova data, with a zero point provided by the disk maser distance to NGC 4258 (Riess et al. 2009). Ultimately, accuracy on the order of $1 \%$ would match accuracies anticipated for $\mathrm{CMB}$ and other data sets in the near future and add a new independent constraint in cosmological parameter estimation. However, achieving this accuracy for $H_{0}$ will be challenging, probably requiring detailed study of a disk maser sample at least 3 times larger than what is available today, assuming maser distances with $10 \%$ accuracy as the norm. The minimum sample recessional velocity would be $10^{4} \mathrm{~km} \mathrm{~s}^{-1}$, though objects as nearby as $3200 \mathrm{~km} \mathrm{~s}^{-1}$ may be useful where peculiar motions are known with uncertainties as small as $160 \mathrm{~km} \mathrm{~s}^{-1}$. Difficulty in accurately estimating peculiar motions in some volumes may restrict this extended sample, e.g., near the Great Attractor or where uncertainty in Tully-Fisher or Fundamental Plane distances are large.

The graduate dissertation by $\mathrm{P}$. K. contributed to this work. We thank M. Elvis, E. Humphreys, and R. Narayan for helpful discussions. This work benefitted from expert analysis of peculiar velocities and model flow fields offered by K. Masters. We thank M. Reid for discussions and the code used to fit the velocity drifts of maser features. We appreciate support and assistance with GBT observing by J. Braatz, as well as overall 
encouragement. We thank C. Bignell for telescope scheduling that enabled the dissertation work, R. Maddalena for in-depth discussion of calibration, and C. Barrett for her close reading and editorial contributions. This research has made extensive use of the NED, which is operated by the Jet Propulsion Laboratory (JPL). This work was supported by GBT student support program, grants GSSP004-0005 and GSSP004-0011, and in part by grant NNG05GK24G from NASA. The results presented here were obtained through NRAO observing programs GBT04C031, GBT05A-015, GBT06A-056 (PI: Kondratko), and AK629 at the VLA.

\section{REFERENCES}

Abazajian, K., et al. 2005, AJ, 129, 1755

Adelman-McCarthy, J. K., et al. 2007, ApJS, 172, 634

Argon, A. L., Greenhill, L. J., Reid, M. J., Moran, J. M., \& Humphreys, E. M. L. 2007, ApJ, 659, 1040

Beers, T. C., Kriessler, J. R., Bird, C. M., \& Huchra, J. P. 1995, AJ, 109, 874

Bottinelli, L., Durand, N., Fouque, P., Garnier, R., Gouguenheim, L., Paturel, G., \& Teerikorpi, P. 1992, A\&AS, 93, 173

Bottinelli, L., Gouguenheim, L., Fouque, P., \& Paturel, G. 1990, A\&AS, 82, 391

Braatz, J., Greenhill, L., Moran, J., Wilson, A., \& Hernnstein, J. 1997a, BAAS, 29, 1374

Braatz, J., Greenhill, L., Reid, M., Condon, J., Henkel, C., \& Lo, K.-Y. 2007, in IAU Symp. 242, Astrophysical Masers and Their Environments (Dordrecht: Kluwer), 399

Braatz, J. A., \& Gugliucci 2008, ApJ, 678, 96

Braatz, J. A., Henkel, C., Greenhill, L. J., Moran, J. M., \& Wilson, A. S. 2004, ApJ, 617, L29

Braatz, J. A., Wilson, A. S., \& Henkel, C. 1996, ApJS, 106, 51

Braatz, J. A., Wilson, A. S., \& Henkel, C. 1997b, ApJS, 110, 321

Braatz, J. A., Wilson, A. S., Henkel, C., Gough, R., \& Sinclair, M. 2003, ApJS, 146,249

Cecil, G., et al. 2000, ApJ, 536, 675

Clarke, C. J., Kinney, A. L., \& Pringle, J. E. 1998, ApJ, 495, 189

Claussen, M. J., Diamond, P. J., Braatz, J. A., Wilson, A. S., \& Henkel, C. 1998, ApJ, 500, L129

Claussen, M. J., \& Lo, K.-Y. 1986, ApJ, 308, 592

Condon, J. J. 2003, GBT PTCS Project Notes (ver. 3; Green Bank, WV: NRAO), 27

Crook, A. C., Huchra, J. P., Martimbeau, N., Masters, K. L., Jarrett, T., \& Macri, L. M. 2007, ApJ, 655, 790

Curran, S. J. 2000, A\&AS, 144, 271

da Costa, L. N., Pellegrini, P. S., Davis, M., Meiksin, A., Sargent, W. L. W., \& Tonry, J. L. 1991, ApJS, 75, 935

da Costa, L. N., et al. 1998, AJ, 116, 1

Darling, J., Brogan, C., \& Johnson, K. 2008, ApJ, 685, L39

Davoust, E., \& Contini, T. 2004, A\&A, 416, 515

de Vaucouleurs, G., de Vaucouleurs, A., Corwin, H. G., Jr., Buta, R. J., Paturel, G., \& Fouque, P. 1991, Third Reference Catalogue of Bright Galaxies (New York: Springer)

de Vaucouleurs, G., de Vaucouleurs, A., \& Nieto, J.-L. 1979, AJ, 84, 1811

di Nella, H., Garcia, A. M., Garnier, R., \& Paturel, G. 1995, A\&AS, 113, 151

Dreyer, J. L. E. 1881, New General Catalogue of Nebulae and Clusters of Stars (1953 ed.; London: Memoirs RAS)

Elitzur, M. 1982, Rev. Mod. Phys., 54, 1225

Erdoğdu, P., et al. 2006, MNRAS, 373, 45

Fairall, A. P., et al. 1992, AJ, 103, 11

Falco, E. E., et al. 1999, PASP, 111, 438

Fisher, J. R., \& Tully, R. B. 1981, ApJS, 47, 139

Fisher, K. B., Huchra, J. P., Strauss, M. A., Davis, M., Yahil, A., \& Schlegel, D. 1995, ApJS, 100, 69

Fouque, P., Bottinelli, L., Gouguenheim, L., \& Paturel, G. 1990, ApJ, 349, 1

Fukugita, M., et al. 2007, AJ, 134, 579

Gallimore, J. F., Baum, S. A., \& O'Dea, C. P. 2004, ApJ, 613, 794

Gallimore, J. F., Baum, S. A., O’Dea, C. P., Brinks, E., \& Pedlar, A. 1996, ApJ, 462,740

Gallimore, J. F., Henkel, C., Baum, S. A., Glass, I. S., Claussen, M. J., Prieto, M. A., \& Von Kap-herr, A. 2001, ApJ, 556, 694

García-Marín, M., Colina, L., Arribas, S., Alonso-Herrero, A., \& Mediavilla, E. 2006, ApJ, 650, 850

Greenhill, L. J. 2004, New Astron. Rev., 48, 1079
Greenhill, L. J. 2007, in IAU Symp. 242, Astrophysical Masers and Their Environments (Dordrecht: Kluwer), 381

Greenhill, L. J., Ellingsen, S. P., Norris, R. P., Gough, R. G., Sinclair, M. W., Moran, J. M., \& Mushotzky, R. 1997a, ApJ, 474, L103

Greenhill, L. J., \& Gwinn, C. R. 1997, Ap\&SS, 248, 261

Greenhill, L. J., Herrnstein, J. R., Moran, J. M., Menten, K. M., \& Velusamy, T. 1997b, ApJ, 486, L15

Greenhill, L. J., Kondratko, P. T., Lovell, J. E. J., Kuiper, T. B. H., Moran, J. M., Jauncey, D. L., \& Baines, G. P. 2003a, ApJ, 582, L11

Greenhill, L. J., Moran, J. M., \& Herrnstein, J. R. 1997c, ApJ, 481, L23

Greenhill, L. J., Tilak, A., \& Madejski, G. 2008, ApJ, 686, L13

Greenhill, L. J., et al. 2003b, ApJ, 590, 162

Greenhill, L. J., et al. 2009, arXiv:0902.4255

Hagiwara, Y. 2007, AJ, 133, 1176

Hagiwara, Y., Henkel, C., Sherwood, W. A., \& Baan, W. A. 2002, A\&A, 387, L29

Hagiwara, Y., Kohno, K., Kawabe, R., \& Nakai, N. 1997, PASJ, 49, 171

Henkel, C., Braatz, J. A., Greenhill, L. J., \& Wilson, A. S. 2002, A\&A, 394, L23

Henkel, C., Peck, A. B., Tarchi, A., Nagar, N. M., Braatz, J. A., Castangia, P., \& Moscadelli, L. 2005, A\&A, 436, 75

Herrnstein, J. R., Greenhill, L. J., \& Moran, J. M. 1996, ApJ, 468, L17

Herrnstein, J. R., Moran, J. M., Greenhill, L. J., \& Trotter, A. S. 2005, ApJ, 629, 719

Herrnstein, J. R., et al. 1999, Nature, 400, 539

Hibbard, J. E., \& Yun, M. S. 1999, AJ, 118, 162

Hopkins, P. F., Hernquist, L., Cox, T. J., Dutta, S. N., \& Rothberg, B. 2008, ApJ, 679,156

Howe, H. A. 1900, MNRAS, 61, 29

Hu, W. 2005, in ASP Conf. Ser. 339, Observing Dark Energy, ed. S. C. Wolff \& T. R. Lauer (San Francisco, CA: ASP), 215

Huchra, J., Davis, M., Latham, D., \& Tonry, J. 1983, ApJS, 52, 89

Huchra, J., Latham, D. W., da Costa, L. N., Pellegrini, P. S., \& Willmer, C. N. A. 1993, AJ, 105, 1637

Humphreys, E. M. L., Reid, M. J., Greenhill, L. J., Moran, J. M., \& Argon, A. L. 2008, ApJ, 672, 800

Hunt, L. K., Malkan, M. A., Rush, B., Bicay, M. D., Nelson, B. O., Stanga, R. M., \& Webb, W. 1999, ApJS, 125, 349

Ichikawa, K., \& Takahashi, T. 2008, J. Cosmol. Astropart. Phys., 4, 27

Ishihara, Y., Nakai, N., Iyomoto, N., Makishima, K., Diamond, P., \& Hall, P. 2001, PASJ, 53, 215

Jones, D. H., Saunders, W., Read, M., \& Colless, M. 2005, PASA, 22, 277

Kameno, S., Nakai, N., Sawada-Satoh, S., Sato, N., \& Haba, A. 2005, ApJ, 620, 145

Karachentsev, I. D. 1980, ApJS, 44, 137

Keel, W. C. 1996, ApJS, 106, 27

Kinney, A. L., Schmitt, H. R., Clarke, C. J., Pringle, J. E., Ulvestad, J. S., \& Antonucci, R. R. J. 2000, ApJ, 537, 152

Kondratko, P. T., Greenhill, L. J., \& Moran, J. M. 2005, ApJ, 618, 618

Kondratko, P. T., Greenhill, L. J., \& Moran, J. M. 2006a, ApJ, 652, 136

Kondratko, P. T., Greenhill, L. J., \& Moran, J. M. 2008, ApJ, 678, 87

Kondratko, P. T., et al. 2006b, ApJ, 638, 100

Koranyi, D. M., \& Geller, M. J. 2002, AJ, 123, 100

Lauberts, A., \& Valentijn, E. A. 1989, The Surface Photometry Catalogue of the ESO-Uppsala Galaxies (Garching: ESO)

Malkan, M. A., Gorjian, V., \& Tam, R. 1998, ApJS, 117, 25

Masters, K. L. 2005, PhD thesis, Cornell

Mazzarella, J. M., \& Boroson, T. A. 1993, ApJS, 85, 27

Middelberg, E., et al. 2004, A\&A, 417, 925

Miyoshi, M., Moran, J., Herrnstein, J., Greenhill, L., Nakai, N., Diamond, P., \& Inoue, M. 1995, Nature, 373, 127

Morganti, R., Greenhill, L. J., Peck, A. B., Jones, D. L., \& Henkel, C. 2004, New Astron. Rev., 48, 1195

Nagar, N. M., \& Wilson, A. S. 1999, ApJ, 516, 97

Nakai, N., Inoue, M., Miyazawa, K., Miyoshi, M., \& Hall, P. 1995, PASJ, 47, 771

Nakai, N., Inoue, M., \& Miyoshi, M. 1993, Nature, 361, 45

Nandra, K., George, I. M., Mushotzky, R. F., Turner, T. J., \& Yaqoob, T. 1997, ApJ, 477, 602

Olling, R. P. 2007, MNRAS, 378, 1385

Osterbrock, D. E., \& Dahari, O. 1983, ApJ, 273, 478

Paturel, G., Theureau, G., Bottinelli, L., Gouguenheim, L., Coudreau-Durand, N., Hallet, N., \& Petit, C. 2003, A\&A, 412, 57

Peck, A. B., Henkel, C., Ulvestad, J. S., Brunthaler, A., Falcke, H., Elitzur, M., Menten, K. M., \& Gallimore, J. F. 2003, ApJ, 590, 149

Pesch, P., \& Sanduleak, N. 1983, ApJS, 51, 171 
Pringle, J. E., Antonucci, R. R. J., Clarke, C. J., Kinney, A. L., Schmitt, H. R., \& Ulvestad, J. S. 1999, ApJ, 526, L9

Reid, M. J., Braatz, J. A., Condon, J. J., Greenhill, L. J., Henkel, C., \& Lo, K. Y. 2009, ApJ, 695, 287

Riess, A. G., et al. 2009, ApJ, 699, 539

Rines, K., Geller, M. J., Diaferio, A., Mahdavi, A., Mohr, J. J., \& Wegner, G. 2002, AJ, 124, 1266

Rothberg, B., \& Joseph, R. D. 2006, AJ, 131, 185

Sandage, A. 1978, AJ, 83, 904

Saunders, W., et al. 2000, MNRAS, 317, 55

Schinnerer, E., Eckart, A., Tacconi, L. J., Genzel, R., \& Downes, D. 2000, ApJ, 533,850

Schmitt, H. R., Donley, J. L., Antonucci, R. R. J., Hutchings, J. B., Kinney, A. L., \& Pringle, J. E. 2003, ApJ, 597, 768

Schweizer, F., \& Seitzer, P. 2007, AJ, 133, 2132

Skrutskie, M. F., et al. 2006, AJ, 131, 1163
Springob, C. M., Masters, K. L., Haynes, M. P., Giovanelli, R., \& Marinoni, C. 2007, ApJS, 172, 599

Staveley-Smith, L., \& Davies, R. D. 1988, MNRAS, 231, 833

Strauss, M. A., Huchra, J. P., Davis, M., Yahil, A., Fisher, K. B., \& Tonry, J. 1992, ApJS, 83, 29

Tarchi, A., Castangia, P., Henkel, C., \& Menten, K. M. 2007, New Astron. Rev., 51,67

Ulvestad, J. S., \& Wilson, A. S. 1984, ApJ, 285, 439

Vorontsov-Vel'Yaminov, B. A. 1977, A\&AS, 28, 1

Wegner, G., et al. 2003, AJ, 126, 2268

Weistrop, D., \& Downes, R. A. 1991, AJ, 102, 1680

Yamauchi, A., Nakai, N., Sato, N., \& Diamond, P. 2004, PASJ, 56, 605

Zabludoff, A. I., Huchra, J. P., \& Geller, M. J. 1990, ApJS, 74, 1

Zhao, J.-H., Anantharamaiah, K. R., Goss, W. M., \& Viallefond, F. 1997, ApJ, 482,186 